\title{
Radioeiement Concentrations in Archean
}

Granites of Centrai Wyoming

by

J.S. Stuckiess, C.M. Bunker, C.A. Bush and George VanTrump, Jr

Open-File Report 81-948

1981 


\section{INTRODUCTION}

The region of central Wyoming (fig. 1) is a major uranium province with several producing uranium districts (Butier 1972). A11 of the current production is from Cenozoic sandstones for which the dominant provenance is nearby Archean granitic terrane (Seeland 1976, 1978). Several isotopic studies have lead to the conclusion that the Granite Mountains lost more than enough uranium to account for that in the surrounding uranium districts and that the timing of that loss was compatibie with the age of ore deposition (Rosholt and Bartel, 1969, Rosholt and others, 1973; Stuckless and Nkomo, 1978, and Ludwig, 1978 and 1979). Seeland (1978) noted that ail of the deposits within the Powder River Basin were situated in arkoses that had been derived from the northern Laramie Range or from the Granite Mountains. Small deposits in the Owi Creek Mountains and the Wind River Range (fig. 1) are within Archean granite or in sandstone derived from Archean granite that crops out oniy a few kilometers away.

Although volcanic detritus is a common component of the mineralized sandstones and has been proposed as a source for the uranium, we consider its significance as a uranium source to be small because it is also a component of the sediments where uranium deposits are unknown. The distribution of uranium deposits seems to be controlied by the Archean granite provenance. Thus, a characterization of the radioelement distribution within the Archean granites of central Wyoming may be usefui in assessing uranium potential in other granitic areas. In this paper we report radioelement concentration data with statistical analyses for samples from the Granite Mountains, the northern Laramie Range, the Hartville uplift, the Owi Creek Mountains, and the Wind River Range. 


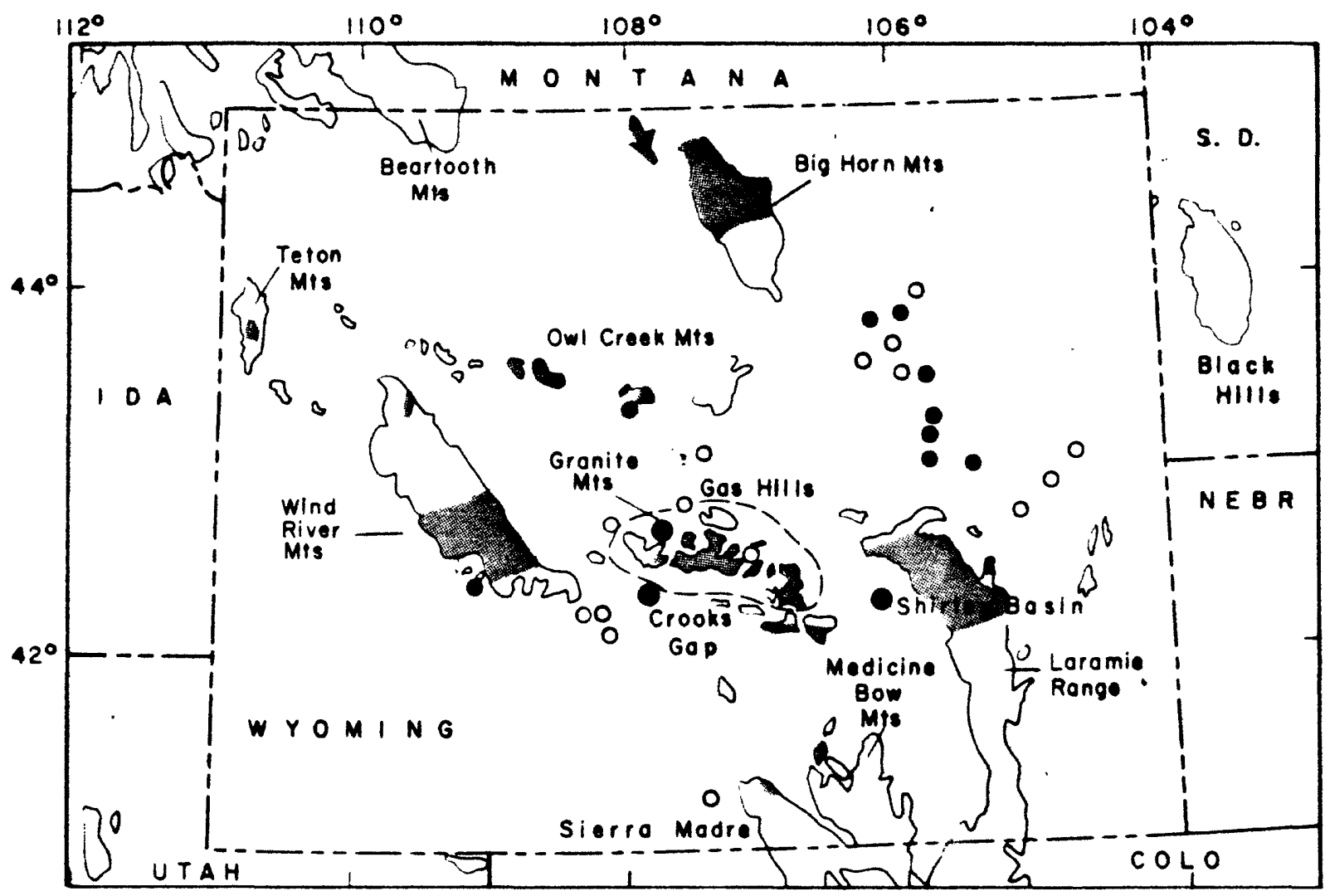

Archeon granites

Figure 1. Generalized geologic map of Wyoming showing areas of Archean rocks and the locations of uranium deposits (circles and dots). The large dots represent uranium districts with reserves plus production of ore $\left(\mathrm{U}_{3} 08>0.1 \%\right)$ of more than 1,000,000 tons. Deposits with ore reserves between 1,000 and 1,000,000 tons are shown by small dots. Deposits with reserves between 1 and 1,000 tons are shown by circles. Uranium data from Butler (1972). 


\section{ANALYTICAL PROCEDURES}

Most of the data used in this study were obtained by seaied-can $\gamma$-ray spectrometry (Bunker and Bush, 1966, 1967). Uranium values determined by this technique are actually radium-equivaient uranium values (RaeU) which represent the amount of uranium needed for secuiar equilibrium with the measured radium. Precision for RaeU and Th is generaliy \pm the quantity 2 percent of the reported amount pius $0.1 \mathrm{ppm}$ absolute, however samples with very high $(>10)$ or very low $(<1)$ Th/RaeU ratios have, poorer precision for the less abundant element. Potassium contents are generaily precise to within \pm the quantity 2 percent of the reported amount pius .03 percent absoiute but again anomalousiy low $\mathrm{K} / \mathrm{RaeU}$ or $\mathrm{K} / \mathrm{Th}$ ratios indicate poorer precisions. A few of the reported uranium and thorium vaiues were obtained by isotope dilution and mass spectrometry (samples $\mathrm{NH}-1-73$ through SB-89H60, tabie 1 from Nkomo and others, 1979; sampies GPA-1 through GPA-13, tabie 1, from Stuckiess and Nkomo, unpublished data). Potassium values for sampies GPA-1 through GPA-13 (tabie 1) were obtained by X-ray fiuorescence. Vaiues for samples BBL-1 through TCMDDH5 have been previously reported by Stuckiess and others (1978), and values for samples 71TDG6 through 72 TDG47 are from Nkomo and others (1978).

All of the samples were collected from outcrops or within a few meters of the surface except as noted in the depth column of tabie 1. At each outcrop, an attempt was made to obtain the freshest material possibie. Exterior surfaces were removed. Nonetheless, all of the samples showed some signs of weathering, including sampies from blasted outcrops, and as much as 25 percent of the more mafic samples from the Granite Mountains are strongly weathered.

Statistical evaluation of the anaiytical data was made by use of the USGS RASS-STATPAC computer system (VanTrump and Miesch, 1977). 
E

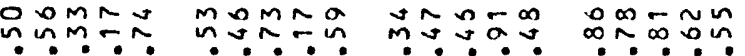
$\stackrel{m}{n}$

$\stackrel{*}{*}$

$0 m n \infty n$

$\because \because \frac{0}{0} \div \div$

$\tau=00$ in

$\because \div \div \div a$

$\sim \ddot{\sim} \sim \dot{0}$

mom:
óami

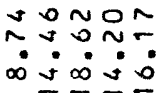

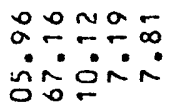

ก̃ $=0 m \tilde{m}$

जिक:

Бํํ응

:ं0mj

응

$\sim 0 \infty \infty$

m $n \circ M N$

¿mina

aN

miñ

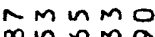

$-\infty a \infty n$

- NN

ivaj:

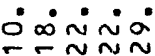

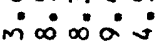

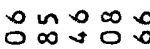

பㄴaㅁ

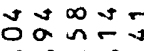

$\infty \sim \dot{0} \dot{0}$

온ㄷㄴ

जिं

on $\therefore 0 \div \infty$

$00 \infty 0 \pi$ $\because m i 0 a$

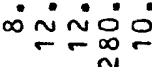

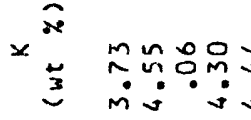

ํำ

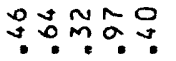

으름

ำกับ

- $\cdots \tilde{N} \infty$ 웅 mijmi

$\bar{n} \sim \widetilde{m}$

กักิ์ mi: in

$\dot{\alpha} \div m \tilde{n}$ min

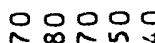

응ㅇㅇㅇㅇㅇㅇㅇ

00000

옹응ㅇㅇㅇㅇㅡ

응응요요

응유옹이

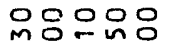

añ

öiñm

añ.

웅ㅇㅇㅇㅇㅇ

inini ษกำง

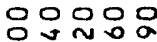
miñ

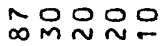

응응용ㅇㅇ mळimin mí் ஸ் ப்ற ini - íni

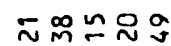 \\ gั̃ก品 \\ जुm}

กำษ

용ำ

$-\underset{\exists}{\sim} m \underset{m}{\infty}$

$\infty 0000$

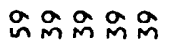

gonom $m m=00$

은드 픈

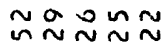
aqmm JMMM $m$ vงa

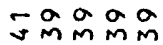
or 드응ㅇํㅇ ิㅡㅇํㅇ응

층ํㅇㅇ

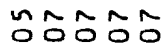

ก̃NN

ñNa

ก̃N

กิก ก

ิㅡㅇํํㅇํㅇ

กีกm狍

$\infty \sim \tilde{m} m$

으순ำ

$m m_{m}+\sim_{m} m$

Un $\sim N=\tilde{N}$

ñजmM

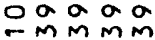

$\ln \ln _{0}^{\infty} \tilde{m}$

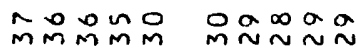

OOOMMM

모ํํㅛ MMOOOO nOOOOO $-\infty \sim \infty \infty$

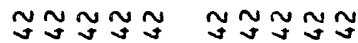

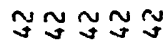

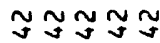

$\mathfrak{v} \mathfrak{\mathcal { v }}$

$\mathcal{M} \mathfrak{N} \mathcal{M}$

$\mathcal{G} \mathfrak{\mathcal { G }}$

$\div \dddot{1}:$ 向的向品

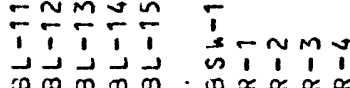

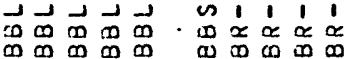

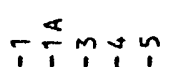
'́⿺廴

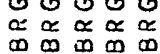

on 0 a

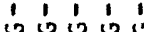

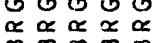

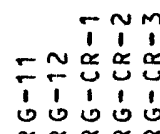

กิ $\tilde{N} \tilde{N}$

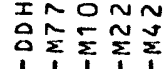

它它

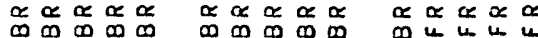

a) o 11111 


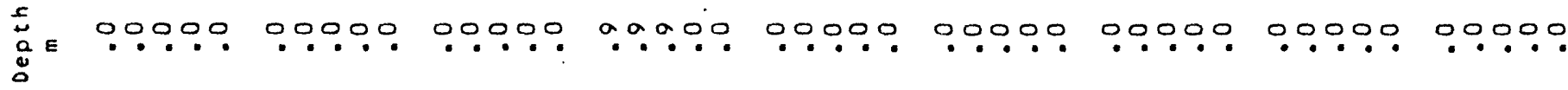

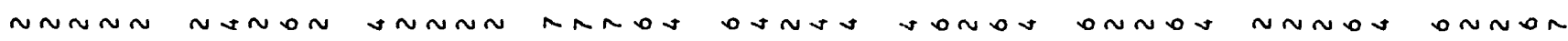

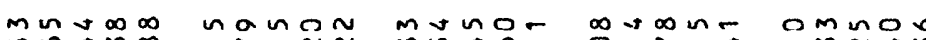
m

$\infty=\infty m-0 m m 0 \infty$ nnovom

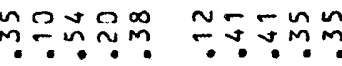
$m \tilde{m} \stackrel{m}{m} \div$

$\circ m m v \infty$ $\because \div$ $\dot{0} \dot{0}$ $\cdots$

กOOO-

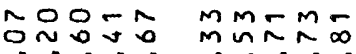
$\dot{m}=\dot{\sim} \dot{\sim} \dot{0}$ in $\sin : \dot{0}$

$00 \sim-0$ $\sim \infty \infty \pi$

$-r+\infty$

íñ

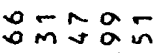

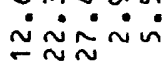

ora in - í⿺辶⿻

D moma

$\therefore \div \div \sim \dot{m}$ $=\prod_{\infty}^{\infty} \sim \tilde{n}$

$\operatorname{sinan}$

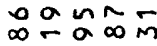
inóm

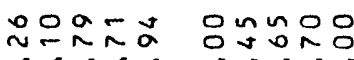

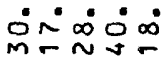

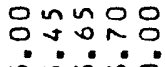
$\infty$ 이요 $\infty m \infty a a$ m n $N$ m
$\Re O 0=\hat{\sim}$ -nádín mona $\begin{array}{ccc}0 & 0 \\ 0 & 0 & 0\end{array}$ oñ⿻n一
ON $\infty$ in ¿mं⿰冫: in $-\sim \sim 0$
$0 \sim, \infty a$ ○一⿻上丨

$2 \approx 0 \div 5$ $\because \div 0=-$ $\sim \sim \infty m a$

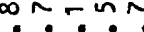
0்
3
$x$
3

워ำ ijiji

0 -ijim

$\exists \tilde{ก} 0 \tilde{ก}$ ijiji

ก⿻上丨n n ……

กำ ¿imim

$\because \infty 2 a n$ نंmin

กิำำ mimin

กำ $=\pi$ in: i

ำ่ำก mimis

上E

은은웅으 은응용으 ¿n-mi 응ㅇㅇㅇㅇㅇㅇㅇㅇ n 숭 $\sim \mp \sim \sim N$ $\sim \sim \sim \dot{\sim}$ บ.ง.․․․ iñom

음옷ㅇㅇㅇ $\because \div 0 \infty \infty$ 요용요 minion

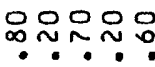
뭏․

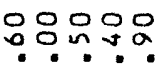
iñ.

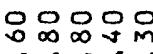
문ำ

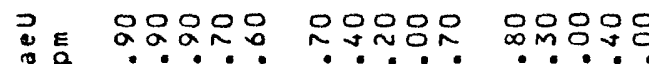

造 inis.

ma iñ?

$\sim m \sim 5 \hat{n}$

$\infty=\sim 2 \infty$

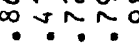

$m_{\infty}^{\infty} \sim \infty$

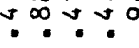

$0 \tilde{0}=n-$

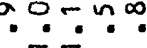

$\infty \div 000$ $\because \because \because m$

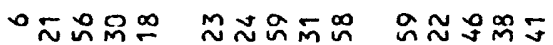
ns u am numain 0000

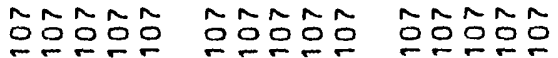

กิำ

moana

MNํํำ

$\stackrel{a}{\sim} \stackrel{\infty}{\sim} \stackrel{\infty}{\sim}$

$\mathcal{U} \tilde{\mathcal{U}}$

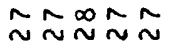

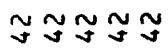
$\sim \sim \sim \sim ⿻ 上$ $\mathcal{U} \underset{\mathcal{N}}{\mathcal{N}}$
업ำ

$m^{\sim} \sim \bar{m}$ in Nㅜㅇㅁㅁ

$\infty \infty \sim \infty a$

รา

$\stackrel{\infty}{\sim} \simeq \div \underset{บ}{0}$

กิ̃ก̃ิ̃

\section{ñNก}

$\infty a 000$

$a \simeq \simeq \mp \cong$

m Navm กิธกิธิ์

ิㅗㅇํํㅇ

ก ก ก M

थ $\bar{m} \bar{m} \bar{m} \sim 0$ 윰ㄴㄴ $\mathcal{I} \mathfrak{\mathcal { Y }}$

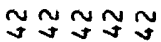
$\stackrel{\sim}{\sim} \sim \stackrel{m}{\sim} \sim$ $\mathcal{H} \mathcal{U} \tilde{N}$ $\checkmark \approx 0 \mathrm{~mm}^{m}$ $\stackrel{0}{\sim} \stackrel{\infty}{\sim} \stackrel{a}{\sim}$ $\mathcal{H} \mathcal{U} \mathcal{U}$ 으으으응

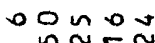
$\sim \stackrel{\infty}{\sim} \sim \infty$ $\mathcal{y} \mathcal{y} \mathcal{N}$
응ํำ $m \infty \sim ⿻ 上$ 드응으음

ปัำin $\tilde{\text { in }}$ ำ $\sim a ̊ a$ $\mathcal{U} \mathcal{G} \mathcal{Y}$ o $=\simeq m \pm$ $\because 0 \pi \infty a 19 \div \frac{1}{1}$

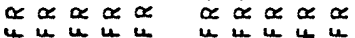

$\simeq 0 \sim \infty a$ $\div \div \div \div$

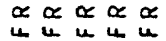

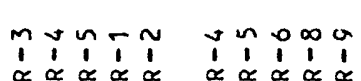

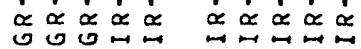

$=\frac{n}{1} \frac{1}{1} \frac{1}{1} \frac{1}{\alpha}$

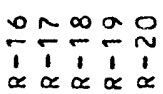

$-\approx m+n$

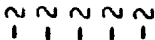

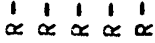

$0 \sim \infty$

N 1ำ 


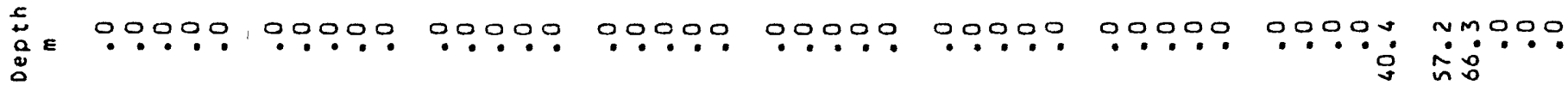

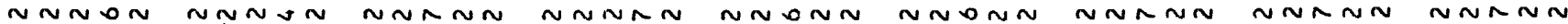

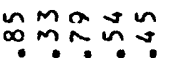

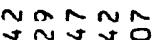

$\because \because \frac{0}{0} \div$

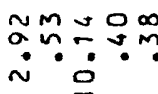

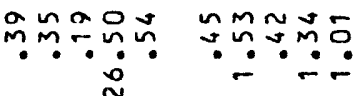

ถุำกัก

$\therefore \dot{n} \cdot \dot{n}$

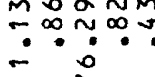

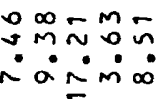

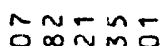

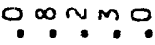

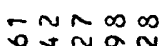

$\stackrel{*}{*}$

$m-i \sim \infty$

$\dot{0} \dot{\sim} \dot{n}$

$\bar{\sim} \sim$

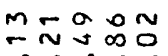

กํㅛㅇㅇ

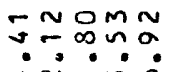

$\dot{n} \sim-n$

옹ํำ

퐁요

$\infty+\infty a$

ดm: $: \cdots$

$\therefore \div \dot{0}=0$

กะด

뭉

$-x^{2}$

inasin

․․․․․

$\simeq \operatorname{ag} \cong$

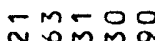

$n \infty m \backsim v$

onmm

ㅇำ

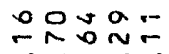

mão

음픙 किं-mio

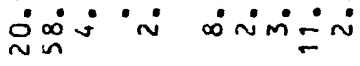

onmino

$\infty \underset{\sim}{\infty} \dot{\sim} \dot{0} \sim \dot{\sim} \dot{\sim} \dot{\sim}$

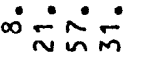

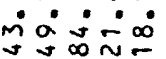

ñن்

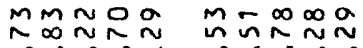
min

$\hat{m}=\hat{\infty}\left\{\begin{array}{l}m \\ \sigma\end{array}\right.$ i.jo: กับำกำ

กั

ำำํำ. anda

웅요

육음응응

언응응우

응응응응

욱우숭ㅇㅁㅇ å̃i்

ヘn் $\dot{\infty} \dot{0}$

$\therefore \dot{\infty} \dot{0}$

융웡ㅇㅇ응 जिiñó 正守的

ơ 0 on

ínis

옹응옹영

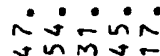

옹은웅응

$\therefore \therefore \dot{m}$

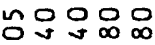

ํำกิ

웅옹요

음ำ

을 ${ }_{\infty}^{\infty}$

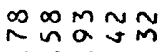

ก- $m \infty$

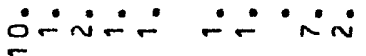

$\therefore \therefore$ iń.

mํㅡㅁㅇㅛ

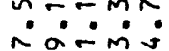

m음옹

isंim-

웅의용

$\therefore$ nín

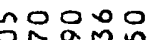
லேm̄ं

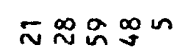

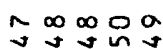

늠ำ

央金我

ヘニニ㤩

오음ㅇํㅇ

ज的星m

ผ워웡ㅇㅇㅝ

N $\approx$ mo

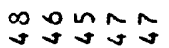

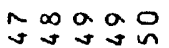

동응응응

응ㅇㅇㅇㅇㅇㅇㅇ

옹옹ㅇㅇㅇ

응옹ㅇㅇ

응ㅇㅇㅇ응

的的高年

응웅용ㅇㅇ

는으.

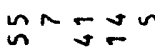

กM⿱

$\simeq$ 는요응

$\stackrel{\infty}{\sim} \div \simeq \simeq$

추ํ응

웅ํำ

$\approx \mathbb{q} \mathfrak{\Psi}$

$\mathfrak{\exists} \mathbb{\exists} \mathbb{F}$
NoOn

Nara

$\sim \sim \sim \sim N$ $\mathfrak{\sim} \approx \mathfrak{*}$
บุำกำ

$\infty \infty \infty \infty a$

-

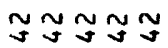

ก ำ

00000

$\sim \sim \sim \sim N$
겅의 엉

约的识品

옹응ㅇㅇㅇㅇ

느응응

은몽

O- 0 No

กำ

$\mathfrak{\exists} \cong \simeq \mathbb{v}$
소응옹워

웅무의

옹응ㅇㅇㅇ

웅요

$\sim \sim \sim \infty \sim$

- $-\cdots$

$\mathcal{\exists} \mathfrak{q} \mathbb{Y}$

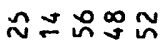
o웅ํำ 웅̃ㅇㅇ

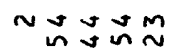
ธ்̃mm $\mathfrak{F} \mathbb{\Psi} \mathfrak{F}$

ก

음 $\sim m$

n 0 - 0 o

으논 נ1

n 0 na 1 J

Oำm $m$

oñ

$\sum_{a}^{1} \sum_{a}^{1} \overline{1}$ 
\& $+\underset{0}{+\infty}$

$\sim \sim 0 \sim$

$\sim \bullet \bullet \sim \sim$

NNNNN

$\sim \sim \sim \sim 0$

$\sim \sim N \sim N$

$\sim \sim \sim \sim 0$

$\sim \sim 00$

on $N \sim$

NONNN

$* x$
$\vdots$
0
0
$x$

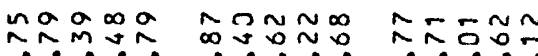

$m \sim \sim \infty O$

nom 0

กลำ

$\because$

i.

$\because 0$

$\sum_{i}^{*}$

กิำ

어요 $m$

$\therefore \infty \dot{0} \dot{0}$

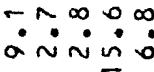

in $0=\pi N$

ก ก ก ก ก

anm

$\because \sim a$ in ?

$\infty \infty \infty \div$

$\sim 0^{\infty} \div$

$m_{\infty}^{m} \infty$.

$0 \dot{0} \dot{n}$

$\because \infty 000$

$\because-\infty \div 0 \%$

$\infty 00 \div 0$

$m=\infty=-$

ํㅜㄴำ

กับง

जिmm:

00500
$0.00 \%$

nan $n \infty$

जo in $\infty$

nं०่

ํำis

$\stackrel{\sim}{\sim} \sim 0$

$\because \div \because \cdots$

$-0 \pm n$

n一ำ요

NONON

$\because 00 \div \infty$

$\simeq \sim \infty \infty m$

$\infty \infty \infty \sim \infty$

$\stackrel{2}{5}$

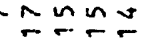

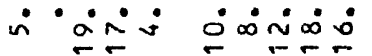

$\dot{\sim}=\dot{\sim} a$

onmon

$\therefore \therefore \infty$ in

i

نே:

$\hat{x}$
$\times$ aेom
$\vec{x}$ inis:

ㅇำ $\sim$ ำ

ำกㅇำ

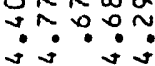

ma에 $\infty$

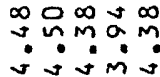

ڤom

ํํํำ

$\div \infty \overrightarrow{0} \div$

ํำำ

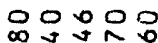

응응으응

응옹ㅇㅇㅇ

응 네잉응

엉ㅇㅇㅇㅇ

은음ㅇㅇㅇㅇㅛ

- $\dot{0} \dot{2} \div \dot{0}$

용요

$\cdot \dot{0} \dot{0}$

응응ㅇㅁㅇㅇㅇ

음은요잉

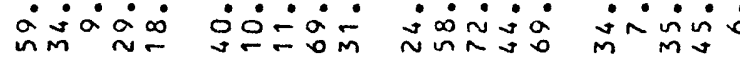

OOMM

$\tilde{0}=\sim_{m}^{\infty}$

a

논

imán

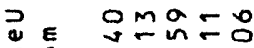

$\propto$ a $\dot{0} \dot{0} \sim \dot{\sim} \dot{m}$

andon

iேn in

웅ㅇㅇㅇㅇㅇ

minisi

$-0 \hat{0} \sim \tilde{\sim}$

$\sim \sim 00 \mathrm{~m}$

응응ㅇㅇㅜ

$\dot{0} \dot{0} \dot{0}$

imin:-

ONMm

00005

$0 \backsim \div 0 \infty$

miñ-

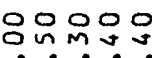

minm-

음 $-\alpha \sim \infty$

m? $\div$ ?

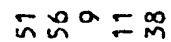

$M \vec{M} M \tilde{m}$

승응응

은ㅁำ

$\sim \sim N \sim N$

MMMMM

$\mathcal{H} \mathfrak{A} \mathcal{H}$

$\hat{n} \sim m \tilde{m}$

$M N \bar{M} O$

ㄱำ 202

ปึฑัต

$a \frac{m}{m} \div$

윰드.

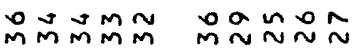

å $\stackrel{\infty}{\sim} \sim \sim n$

aํำก

nañ

nñ

กnN

nñN

ñNA

응으응

$n$ nn $\operatorname{nn}^{2}$

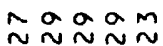

nNNR

응으응

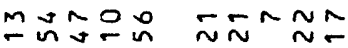

ก

OONEMM

$N-N N$ in

MMMMm

MMMMM

$\underset{\mathcal{G}}{\mathcal{G}} \mathbb{G}$

$\mathcal{A} \mathfrak{N} \mathbb{Z}$

ヘヘ ํํำ

$\simeq \approx \sim m a$

mล̃ก̃

NOOOON

กヘกヘ

응으응

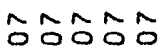

ogrino

nMN $\sim$ Mmmmm

$m \backsim a \sim 0$ $\tilde{G} \mathfrak{G} \tilde{G}$

$\mathfrak{q} \sim \mathcal{N}$

$\sim \sim \sim \sim \sim ⿻ 上$

$\mathcal{N} \mathfrak{N} \mathcal{G}$

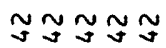

$\mathfrak{q} \mathcal{N} \sim$ $\because 0 r \infty a$ 10.5
OFNmv

$\div \div \div \div \div$ 잉ํㅇ n 0 - $\infty$ a

品

$1,157.0 N m v n$

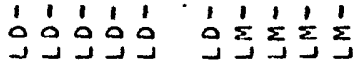

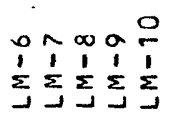

$\because \approx 000$

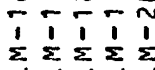

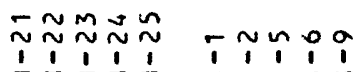
$\frac{1}{\Sigma} \sum^{1} \sum^{1} \frac{1}{\Sigma}$ is is is is 
f

$\stackrel{+}{a}$

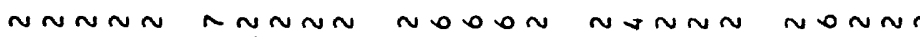

Nonn nNon

nกง $n$ NonN

$\stackrel{*}{*}$

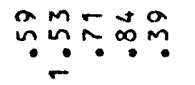

$\sim-N \infty \infty$

n-mos - nom

ñagn

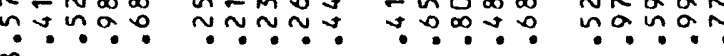

ㅇำ

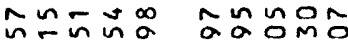
$\stackrel{\infty}{m}$

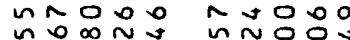
aiำ

i்:

$\infty a+\tilde{\sim}$

*

mianis

$\operatorname{rina0}$

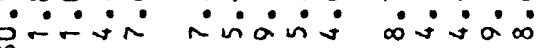

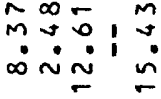

$\infty \sim n \infty a$

$\because 0.90$

움ำ

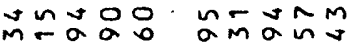
क-

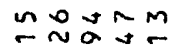

ํํำ융ㅇㅇ

영요의

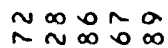

añ

$\because \dot{0} \sim \dot{a}$

$\therefore \dot{m} \div \dot{0}$

Nádi

$\therefore \infty \div \div \dot{0}$

ல்ட்-

05010

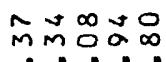
$\ddot{\sim} \div \sim \infty$

กํㅇํ요 ¿niva

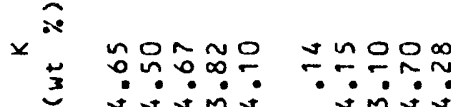

ă

$00 \div \div \frac{1}{4}$

MN

$\because=\div \simeq \infty$

$\approx \tilde{\infty} \infty, \stackrel{\square}{0}$

$\sim \infty \sim ⿻ 上 丨$

$\because \infty . \div \div$

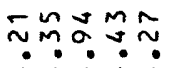

กัง

inidi imiji

\section{上}

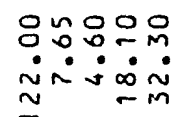

응옹으요의

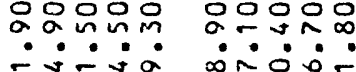

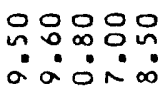

옹ㅇㅇㅇㅇㅁㅇㅛ

응용ㅇㅇㅇㅛ

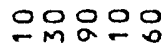

mã

maำ

NmEก

mãn

$\because \div \div$

$\sim \underset{\sim}{\sim} \tilde{m} \tilde{n}$

영용요

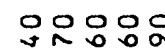

영잉응

엉용요

MOONR̃

임

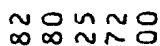

ivimi

जिं:

miñin

언음용요

은응요응

iñí: jminis

$\tau^{m} \sim^{m} 0$ $\because \simeq \div \infty \div$

ำำ

$\sim \bar{n} \sim ⿻ 上$ 융요

ํํํํํํำ ํㅡㅇㅡํ응

คิกิธีก

กNAN

응으응

กั

$\operatorname{ng} \min$

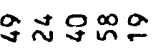

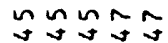
toga

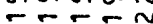

응ㅇㅇㅇㅇㅇㅇㅇ

응응ㅇㅇ응

ํํㅇํ응

mov $v$

OM $N o$

$-\sim \backsim n ̃$

$\tilde{M} \sim \dot{m} \tilde{N}$

$-\sim m m m$

vin $-m$

$\checkmark M M N \sim$

$\sim M M \sim M$

음을

mimmin

$\mathcal{H} \mathcal{Y} \mathcal{Y}$

$\stackrel{\sim}{\sim} \underset{\sim}{\sim} \sim$

$\sim \sim \sim \sim \sim ⿻$

$\mathcal{G} \mathcal{G} \tilde{\mathcal{G}}$

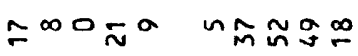

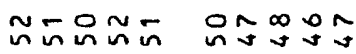

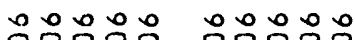
으으으으음은으은

仙少 $M \sim \infty \infty \infty \infty \infty \sim 0$ $\sim \sim \sim \sim \infty$ ก $\sim \sim ⿻ 上$

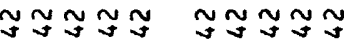

15

은

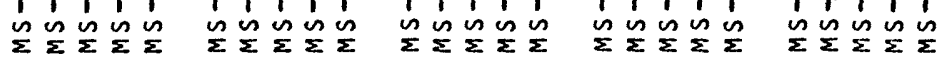

imin a $\begin{array}{llll}0 & 0 & 0 & 0 \\ 0 & a & 0 & 0\end{array}$
$0 \sim \infty a \frac{0}{1}$

1'1 11 ' â $\div \div \frac{1}{1} \div \frac{1}{1}$

o $\begin{array}{llll}0 & 0 & 0 & 0 \\ 0 & a & a & 0\end{array}$
느요

1'1 1 1

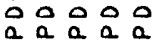


ह $\stackrel{+}{i} \underset{0}{i}$

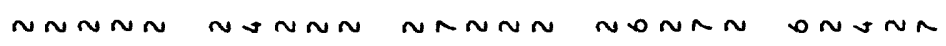

ก ก ก ก

ก.ก ก ก

$\cos$

$\checkmark \sim \sim N \sim$

mmõ丶

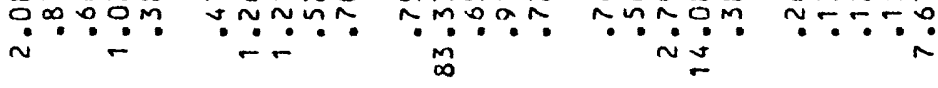

$\checkmark \hat{M}=\tilde{N} M m m i n$

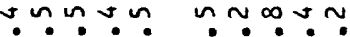

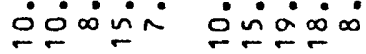

จกิธกับ

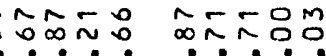
$\simeq \therefore 0 \div \dot{0}$

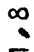

a $00 \infty$ on

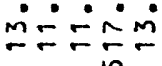
íं $\dot{\sim} \dot{0}$
OM요 $\because ? m \cdots \infty$

$0 \sim \bar{m} \tilde{0}$ in

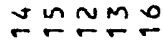

$v=-m \infty$ $\because \simeq ? \cap m$ $\infty \dot{0}=$ $m \sim m a O$ i. in is

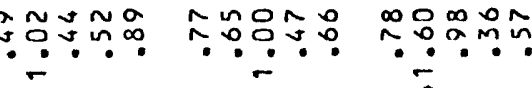
Doñ : imo
둥응 जini⿻․ ธ에 ñ⿻上丨-

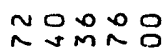
○் $0 \dot{0}$ กำㄴำ क0ं $\dot{0}$ กษัง - $\sim \dot{0} 00$

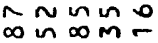
in iोa $\operatorname{mos}$

$x$

음 $m \tilde{n}$

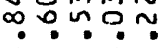

음ำ

imivi

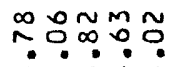

i. mi i

ำง

máa 幺 ma:

$\stackrel{0}{0} \div=\stackrel{0}{*}$

응 过过

으ำ

-áñ

ง - $\dot{0}$ i :

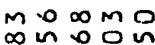
$\infty$ in 0 on m ñ

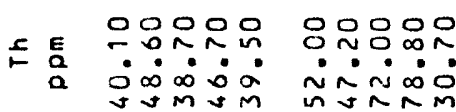

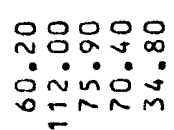

엉응으응 mím n

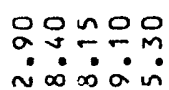
$\simeq \infty \infty a n$
영웅응ㅇㅁ $\therefore \sim \dot{\sim} \dot{0}$ กิำ ำ
응용요 min: monsm

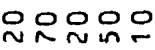
0. - :

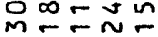

은응오오 mำ minio $\sim m \sim ⿻$

응응ㅇㅇㅇㅇㅇ

$m \sim a m \infty$ نேn்

งกำ iับำ

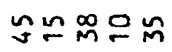
$\sim \infty \infty a \sim$ 2avt

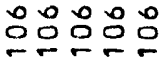

ing on nnom $n$ NNNNN

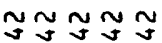

in $\sim \mathcal{\sim} \sim \mathbb{N}^{5}$ $\checkmark n \sim 0 m$ $\mathcal{H} \mathcal{M} \mathcal{T}$

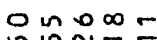
$\infty \cup \sim n$ $\sim \sim \sim \sim N$ ก⿻ำ

duำ $\sim \sim \infty \infty$ in $\sim \sim N \sim N$ $\mathcal{y} \mathfrak{\mathcal { U }} \mathcal{U}$

$0-n m$ - Nm 1!1! aaaa on $\sim \mathfrak{i}$ N $\begin{array}{lllll}1 & 1 & 2 & 2 \\ 0 & 0 & 0 & \alpha & \alpha \\ 0 & 0 & 0 & 0 & 0\end{array}$

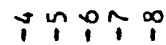
$3 z \frac{3}{2} z$

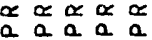

$9 \div 5 \div 5$ $33 \frac{3}{2} 3$ 웅 중 웅 a ง 잉. 00 $\sim \sim-5-$ $\mathcal{U} \mathfrak{\mathcal { Y }}$

$m \sim a \sim \infty$ $\infty \wedge \sim \pi$ rarn

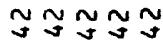

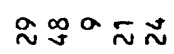

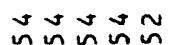
웅유 웅ㅇㅇㅇㅇㅇ ñññ

○० 000

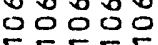
$\operatorname{sind}$

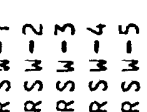
$\propto \alpha \propto \alpha \alpha$ a a a
ำ

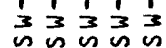

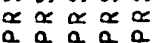

$m=\infty 0 \mathrm{~g}$ ํำกำ

웅ㅇㅇ

응ㅇㅇ은

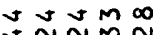

- N-ON

$\sim N N \sim N$

$\mathcal{T} \mathfrak{\mathcal { Y }}$
봉ํำ

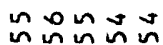

응응웅응응

ornus a $000-$ rata $\mathcal{A} \mathcal{N} \mathcal{N}$

$\sim m \leq \ln 0$ 후눈 的的的的

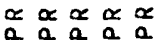

- บMบำ 10.11 11111 으요용 in is in is is 
a $\stackrel{+}{a}$

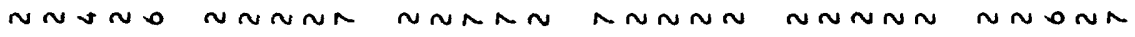

○๐

$\operatorname{sun} 2$

$\checkmark 0 v \sim N$

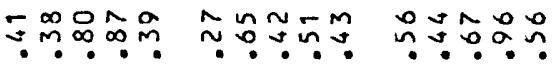

$\because \infty \infty \sim ⿻ 上$

moñm

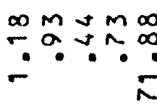

on $\infty i n$ $a$

ONmUn $N-\infty n N$

$\because a m$.

$\approx \sigma N=\approx$

$\therefore \dot{0} \sim \dot{m}$

$\ddot{m} \dot{0} \div \underset{0}{\infty}$

$\because \div \div \div$

Nom

(n)

in

ㄴ.?

$\infty=00 \infty$

$\ddot{0} \cong \dot{0}$

$\dot{0} \dot{m} \dot{0}$

$\therefore \ddot{0} \dot{0} \dot{0}$

$\infty \sim \infty \cup$

\%on

ñmin

$0 \infty a=0$

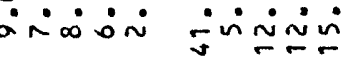

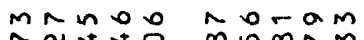

응ㅇㅇㅇ

$\hat{m}-\infty \infty i n$

잉요

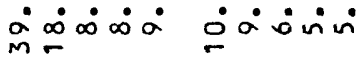

ำกำ

iñ்

며

0 oñ

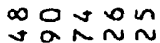

$0 \sim a \circ m$

ก

머ำ

$\because m i \dot{\sim}$

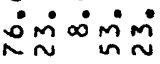

$\stackrel{3}{x}$

$5 \stackrel{\infty}{0} \div 0 \%$ 음

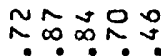

ㅎํำㅇำ

응요은

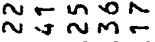

iिim

ivimim

minं:

जिंजिं

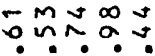

บัน $\dot{m i n} \sim$

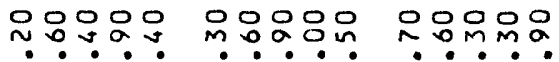

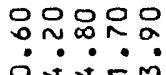

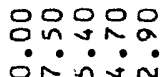

은응ㅇㅇ응

잉ㅇㅇㅇㅇㅇㅇ

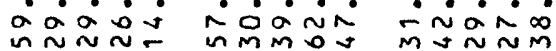

mũñ

oñun

ํㅝㅁ웅

-am-i

엉잉요

imimia

응응ㅇㅇㅇㅇㅇ

maz-

$m \rightarrow \infty$

$\sim m a \sim \infty$

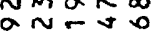

0

no 00 N

00000

음ำ

ก ก ก

ง

응ㄷㅇㅇㅜ

nom

웅잉영으

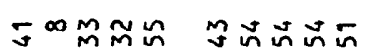

Nmañ

and 0 ing

ป⿻

mก๊ $\approx N$

กั

gำกำ

ơ 000

in in $0 \pi$ in

웅ㅇㅇㅇㅇㅇㅇㅇ

ํํㅇํํㅇํㅇ

in in 0 in

ongaga

동ํํ웅

등으응

ํㅜㅇㅇㅇㅇㅇㅇ

웅ㅇㅇㅇㅇㅇㅇㅇ

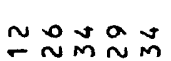

뚠요

$\stackrel{a}{n}=0 n$

$n-\tilde{n} \sim m$

$\because \pm 000$

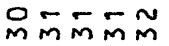

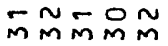

MMM

$\mathcal{N} \mathfrak{N} \mathcal{N}$

$\mathfrak{N} \mathcal{N} \mathfrak{N}$

$\mathcal{G} \mathcal{\Psi} \mathcal{Y}$

$\mathfrak{N} \mathcal{N} \tilde{q}$

$\leq \infty$

$\underset{\substack{0 \\ \text { E } \\ 0 \\ \text { is }}}{\stackrel{0}{0}}$

ำกำ is is is is is $\sum_{n} \sum_{n} \sum_{n} \sum_{n} \sum_{n}$ $\sum_{n}^{1} \sum_{n}^{1} \sum_{n}^{1} \sum_{n}^{1} \sum_{n}^{1}$

T⿻y𠃋? 㟧㟧㟧

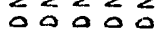
is in in in is is in in

$\div \frac{1}{1} \div \frac{1}{1}$

㟧崖崖

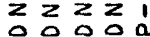
的的被 n n $\stackrel{\infty}{\sim}$

$20 \infty a$

오ํํํํํำ

은 요의

응ㅇㅇㅇㅇㅇํㅇ

ลิ人̃̃

응으으으음

$\stackrel{m}{\sim} \sim \bar{N}$ in

voy ing a $\cong \tilde{N} \tilde{N}$

$\mathfrak{N} \mathcal{N} \mathfrak{G}$

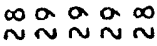

$\mathcal{N} \tilde{N} \mathcal{N}$

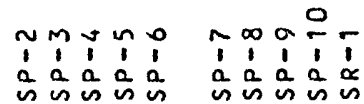


Е

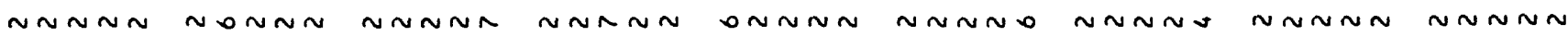

moñ

으요

$\approx \sim \sim \sim N$

$m-\sim N \tilde{m}$

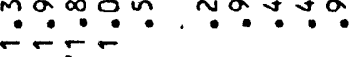
$\sim$

บำ?

I moo mo in

$\sim \sim 0 \sim N$ $\because \because \frac{\infty}{2} \div \div-$

$\infty$ in $\infty$ in

mo $0=0$

$\sim m \infty \sim$

$m o n g m$

gón $m a$

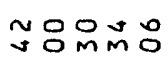

$\because \div \because 0$ $\sim \dot{0} \dot{\sim} \dot{0}$

inín

$\approx \approx \sim \pm 0$

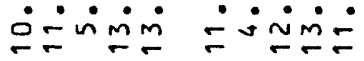

inimi:

oํํㅁ

í⿴囗十்

$\infty-\infty \infty \bar{\infty}$

ن iñ

od nnm

naraga

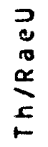

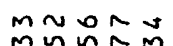

DanOO

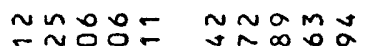

ஸ்ன்

ஸ் $\div \dot{0}$

$\ddot{\sim} \dot{\infty} \dot{\infty} \dot{-}-$

نே்

$\sim \sim m \sim m$

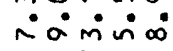

$0 \infty-5 n$

$\because \therefore m-i$

mon 0 a

imisi

으요요

r.r.

$\sim 2+\infty$

m-

$\underset{3}{\pi}$

mañmำ măำ

ำำำ

은 $\simeq a \infty$

जिษ

ำ? ำ?

บฺุ

जिimini

जिmin

$\because 0 ั 5$ ก

mimis:

I $\approx 000$ $\checkmark v N m$

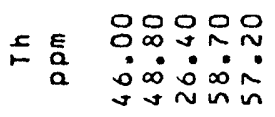

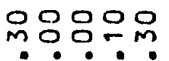

옹응옹오

은응ㅇㅁㅇㅛ

은용유

응윤윰은

ํำ

$\sim \dot{\sim} \dot{0} \div \dot{0}$

$\therefore-i a r$

$=\because \infty \dot{N}^{\infty}$

음웅

응은운은

iñan

ammñ

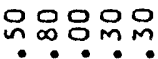

$\because \sim \dot{0}$

음융요요

-

ㄷำ

í⿴囗十

0요요

이응

00000

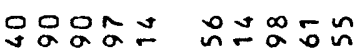

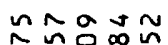

o oono

A-nno

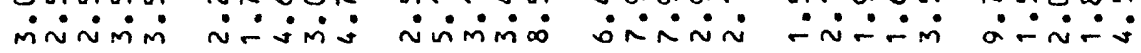

min:-

$\sim \div 0 \div$

OMmo̊a

$\dot{0} \dot{n}-\dot{0}$

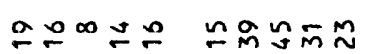

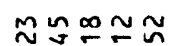

mํํํำ

กำ

in $\mathcal{n} \approx 0$

o ำ ำก

mo믄

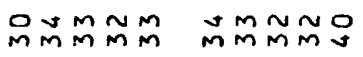

o

กิ

กิ $O \stackrel{\sim}{\sim} \bar{m}$

mmann

ํㅡ으으음

ิㅡㅇํํ응

ํํํํํㅇ

กิธิกี

ิㅡㅇํํํำ

ํำกำกับ

ำํำำำำ

N $\sim$ 的

$\sim \tilde{M} M \dot{m}$

$\approx$ 응 $\bar{m}^{\infty} \bar{n}$

nnunn

m与ñ

$n-\sim \backsim N$

$\infty m v 0 s$

0
0
$\vdots$
$\vdots$
$\vdots$
0

a. 00

ms - v

ง会正品

n $m \sim \infty 0$

ํํำ哽

承瓷兄

운인

- moa

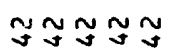

$\tilde{U} \tilde{\mathcal{U}}$

$\mathfrak{U} \mathcal{G} \tilde{\mathcal{V}}$

$\underset{\mathcal{G}}{\mathcal{U}} \mathcal{G}$

으노

$\sim \infty-\infty \sim$

Nmoo in

mOM

o $\infty \backsim \sim 0$

onmon

$\mathcal{H} \mathcal{M} \mathcal{Y}$

$\mathcal{M} \mathcal{U} \mathcal{U}$

$\tilde{\mathcal{N}} \mathcal{N} \tilde{\mathcal{U}}$

in $\mathrm{n}^{\infty} \approx \infty$

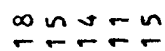

gn

m폰

$\stackrel{v}{a}$
E
0
is

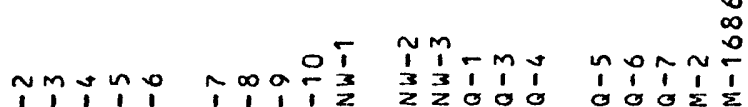

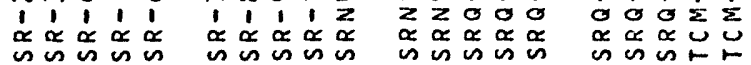

용ㅇㅇ

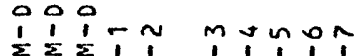

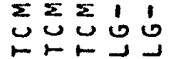

$\infty 0 \div \div \frac{1}{1}$

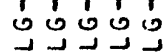

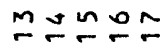

1: $15 \div$

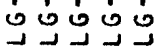

ma윰 $\div \overline{1} \div \tilde{1}$

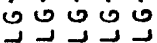


音

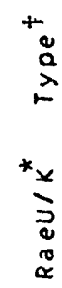

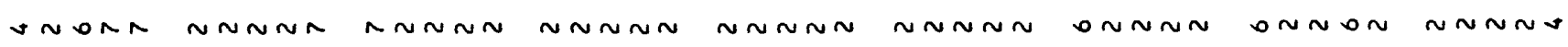

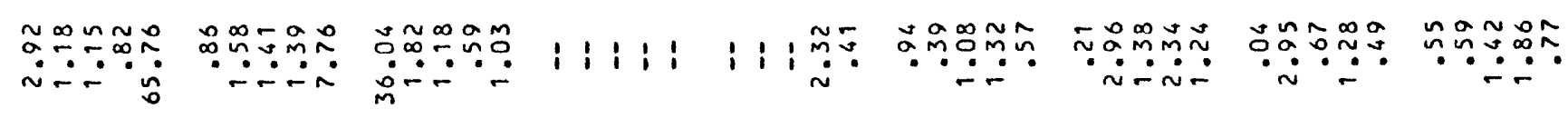
$\checkmark \sim m \sim \infty N N-\infty N-\backsim v 0 \infty$

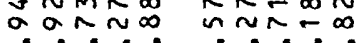

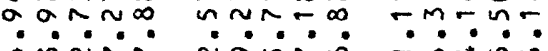

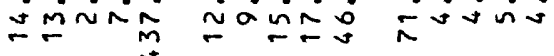

1:1:1

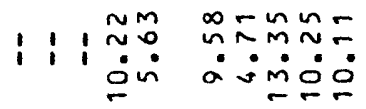

$\alpha= \pm \infty$ annus. onmon

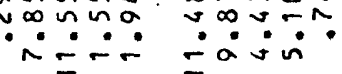

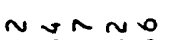

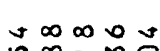

nadon

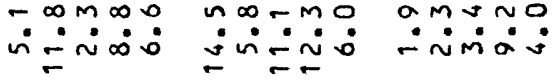

mu-En mmmom

$\sim m \sim 0^{\circ}$

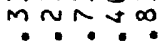

$\div \infty \sim \sim \infty$

으누응

$m \circ \approx=0$

$0 a=n 0$

$\rightarrow 000$

¿inin

imicia.

inin:

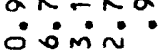

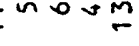

$-\infty$

$\frac{2}{3}$

$\bar{m}= \pm \sim m$

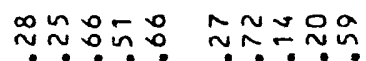

1111:11: 骂

- 980 요

nn $\operatorname{nn} m a$

mingi:

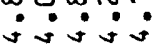

음드.

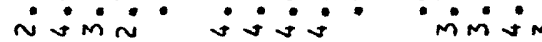

i⿻imi

ininim

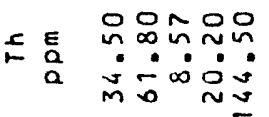

잉웅요음

웅응응

Oลำm

mㄷำ

응ㅇㅇㅇㅇㅇ

máx $\dot{0} \dot{0} \dot{0} \dot{0} \dot{0}$

vájó

ก⿻ำ.ㅇำ

minisio

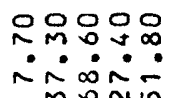

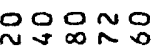

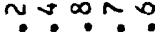

过守的

응ㅇㅇㅇㅇㅇㅁㅇㅛ

umanm orin

rmarn

in $m$ in $m$

monin

ํำ요

ว

mกำ

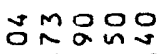

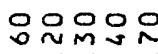

응응옹으

mívín

$\therefore \therefore \dot{0} \dot{0}$

있옹응응

ถะก

\& a Dimin

mi००ण

$\infty \infty \div-\dot{n}$

mंே்

$\because \sim \dot{\sim} \dot{0}$

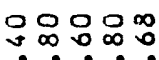

ín் $\dot{\sim}$

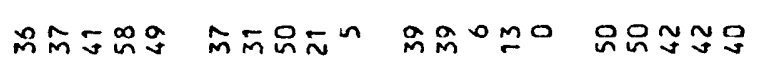

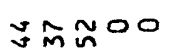

00000

00000

$\hat{\sim} \stackrel{\infty}{\sim} \widetilde{\cup}$

in

กำ识一

家是示过

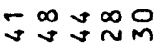

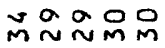

오ำ经法

$\bar{m} \mathfrak{\sim} \simeq \bar{n}$

ํำ ำกำ

经经经品 ำกำํำ

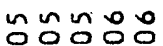

经起经品

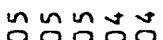

可证过

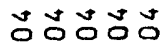

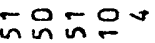

- 0 으는

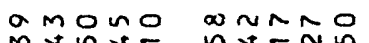

$\infty \infty \infty \sim 0$

mminn

$\frac{2}{2} \ln ^{2}$

央战合

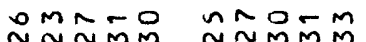

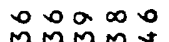

$\bar{\sim} \tilde{\sim} \approx$

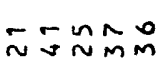

00000

00000

$\sim \infty \sim a$

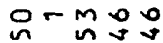

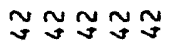

$\mathfrak{\exists} \widetilde{\exists} \widetilde{\exists}$

$\mathfrak{\mathcal { Y }} \mathfrak{\mathcal { Y }}$

$\mathcal{F} \mathfrak{\mathcal { F }} \mathfrak{\exists}$

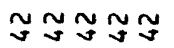

$\hat{M} \cong \mathscr{M} \tilde{M} \hat{m}$

Mำกำ

$\stackrel{\sim}{\sim} \sim \tilde{N} \sim$

oanas

$\mathcal{U} \mathbb{N} \mathfrak{N}$

$\mathfrak{U} \mathfrak{U} \mathbb{J}$

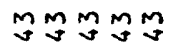

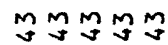

mํำ

象品的留

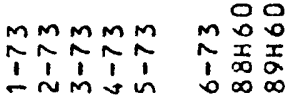

IิTTT

in

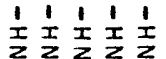

0 $\infty-n$

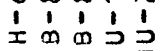

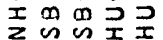

mํํำ年

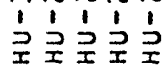

$\stackrel{\infty}{\sim} \backsim \mathfrak{m} \div$ $\because \because 1 \div 5$

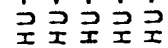

$\sim m 00$

언ㄷํㄴ 영ㅇㅇ

든
- NUn

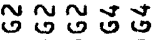

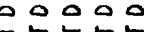
-5ะñ 
点

$+\frac{1}{2}$

$\stackrel{2}{2}$

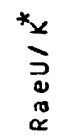

$\underset{5}{*}$

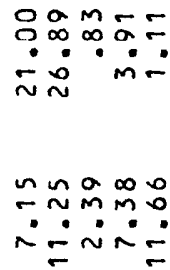

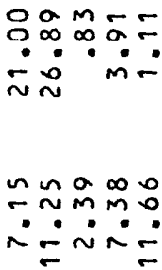

00000

Non

Niñ

a $M N$

$n$ in $v$

$m-n$

กำ

ำ?

$\stackrel{\infty}{m !} \div a$

$\tilde{N} \tilde{N} N$

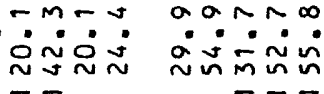

On-no

00000

$\because$ in 0

-

$\circ \sim \infty \sim m$ N ก a. กON

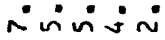
inimini
N $N$ a mñ் m人nva - $0 \backsim 0 v$ a두두
ป⿻一𠃋ำ inmi-
응요 $x_{\infty}^{\infty} 0 \hat{n}$

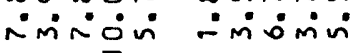

$\underset{3}{3}$

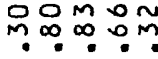

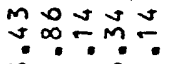

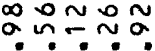

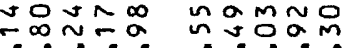

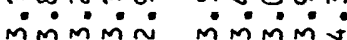

ํำกั่งกำ

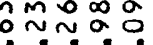
minisis mismis

$\operatorname{mon}_{0}^{0.0}$ जinं-

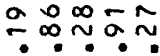

웅응음 ن iñ:

응옹유운. $\dot{0} \dot{0} \dot{0}$

응옹웅요 $\dot{\infty} \dot{\infty} \dot{0}$ $\sim \infty 0 \infty \sim$

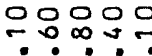
nंنेa nomso 웜ํำ Nonm

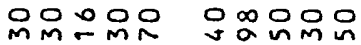
विं்́ं m.

우늄ㅇํㅁ ini-: ง. $\because \dot{n} \dot{0}$

능영용응

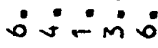

00000 m. - in

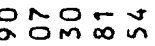
inj:

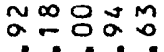
$\stackrel{\sim}{\sim} \approx \sim \infty 0$ ara
มै

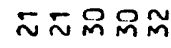

뜨늠음요

ำกำกล กำ品的的 모슨 ิㅗㅇํㅇํㅇㅇㅡ กิ̃ก̃ กับนทีนก

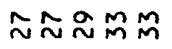
$\sim \sim \sim \sim N$

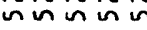
ñ̂̃̂́ กิกิ̃ก̃ 으응ㅇㅁ응으

스응ㅇํ응 응ㅇㅇㅇㅇㅇㅇㅇㅇ

드윰ำ

$\because \backsim m \sim 0$

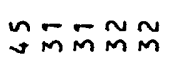
Noñming assan 志足主 mañ

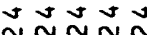
证出出

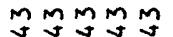

ป气⿵人一

它苟

o-

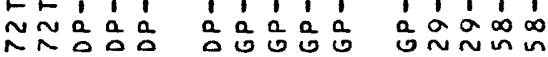

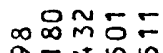
omin inn
음ํำ

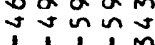
miñ
可的部落 $\sim \sim \sim \sim \sim$

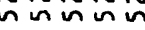
$\tilde{m} \tilde{m} \tilde{m} \tilde{m}$
00000 $m \backsim n m o$ aaga 으우응ㅇㅇ

00000 웅요 $\mathfrak{*} \approx \widetilde{*}$

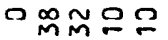
IN $0 \mathrm{Mm}$ 옹응ㅇㅁ응

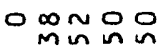
ํㅜ웜 $\approx \approx \approx \sim ⿻ * 乛$ m스능응

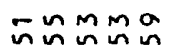

$\infty \infty \infty \infty \infty$

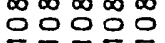

in $000 \mathrm{~m}$ 둥ㅇㅇ $\mathfrak{\exists} \simeq \widetilde{\exists}$ 오ำㅇํ워 冓-mú $\infty 00 \infty$ 으으오옹으

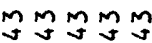

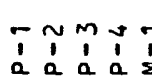
a a a a

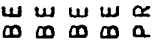

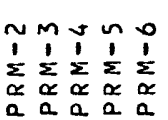

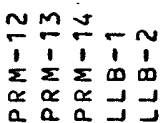




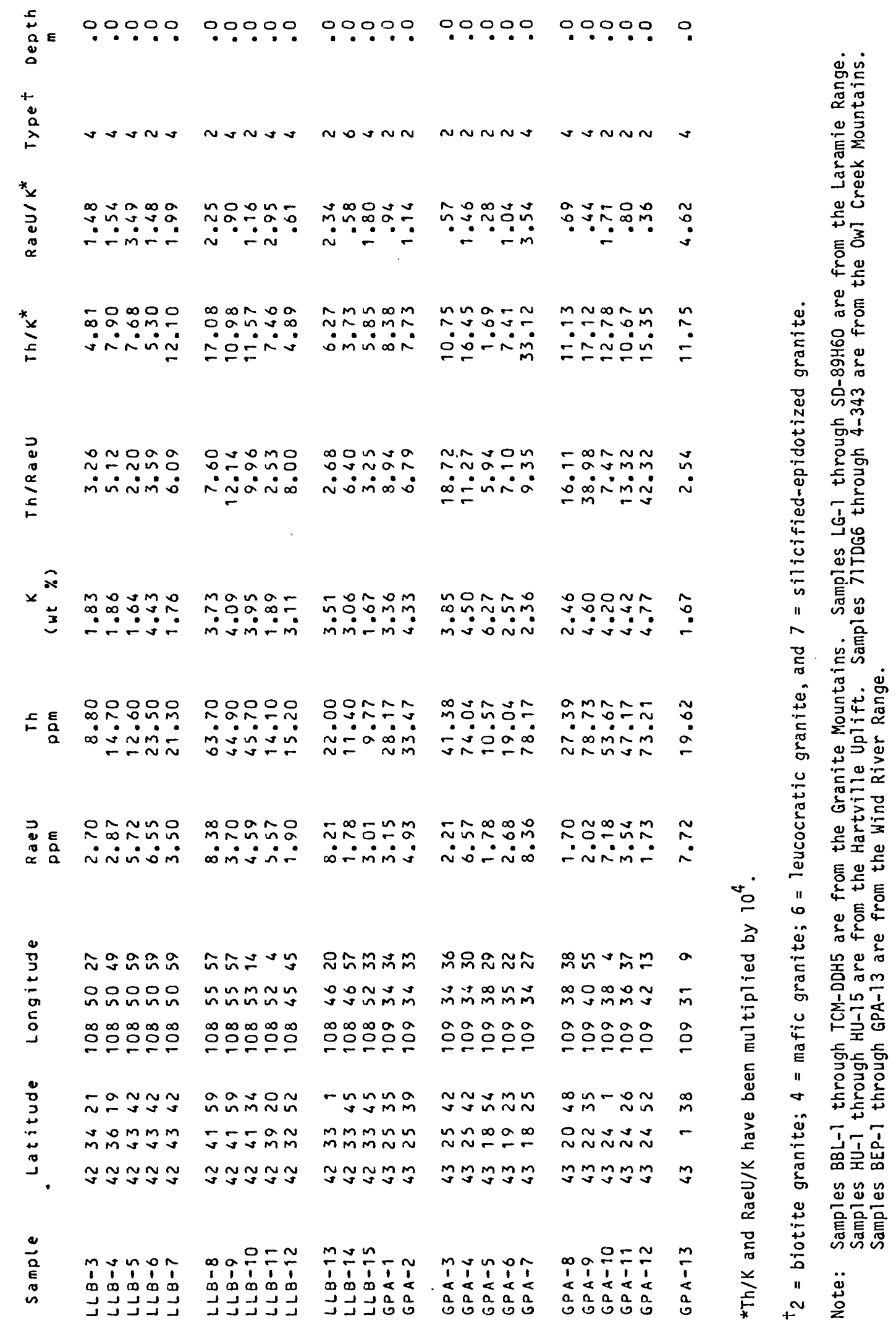




\section{GEOLOGIC SETTING}

The geologic setting is similar in the five areas studied. In each area the granitic rocks were intruded into a metamorphic sequence of amphiboiite facies. The metamorphic sequence is heterogeneous but is dominated by metasedimentary rocks with graywacke as the most common precursor (Peterman and Hildreth, 1978; Condie, 1969; Snyder, 1980; Tourtelot, 1953; Thaden, 1976 a,b,c; Pearson and others, 1971; Granger and others, 1971). Few radiometric ages are availabie for the time of metamorphism, but ages of approximateiy 2,900 m.y. have been reported for the metamorphic rocks in the Granite Mountains (Nkomo and Roshoit, 1972; Peterman and Hildreth, 1978). Somewhat younger ages have been obtained for metamorphic rocks from the Owi Creek Mountains (C. E. Hedge, oral communication, 1980), from the Wind River Range (Bassett and Giletti, 1963), and from the Laramie Range (Johnson and Hilis, 1976), aithough the latter area aiso yieided a tentative age of $2,960 \pm 220$ m.y. for a silitimanite - bearing gneiss ${ }^{1}$.

In the Granite Mountains and the Wind River Range, granitic rocks were emplaced during at least two postmetamorphic, intrusive episodes (Ludwig and Stuckiess, 1978; Nayior and others, 1970). The northern Laramie Range may aiso be formed by more than one Archean granite (Wenner and others, 1981).

Ali of the intrusive ages for areas sampled for this study are late Archean except for those of the Hartvilie uplift where some middle Proterozoic granite is reported (Snyder, 1980). In the Granite Mountains, the volumetricaily minor granite of Long Creek Mountain has been dated by the U-Pb zircon method at 2,640 $\pm 20 \mathrm{~m} \cdot \mathrm{y}$. (Ludwig and Stuckiess, 1978). An estimated 85 percent of the Granite Mountains is made up of the biotitic phase (biotite

1 Where necessary, ages cited in this paper have been recalculated using the decay constants recommended by the IUGS Subcommission on Geochronology (Steiger, and Jager, 1977). 
2 to 15 percent by volume) of the granite of Lank in Dome. Another 10 percent is made up of the ieucocratic phase of this unit. The granite of Lankin Dome has been dated by the U-Pb zircon method at 2,595 $\pm 40 \mathrm{~m} \cdot \mathrm{y}$. (Ludwig and Stuckiess, 1978), and the biotitic phase has been identified as the major contributor of uranium (in terms of $g$ U loss per gram of rock) to the surrounding area (Stuckiess and Nkomo, 1978).

At least two major granitic units crop out in the Wind River Range: the Louis Lake batholith of Bayley (1965 a,b) which is a quartz diorite to granodiorite, and the Popo Agie batholith of Pearson and others (1971) which is a granite and quartz monzonite. The Louis Lake bathoiith has been dated by the U-Pb zircon method at 2,642 $\pm 9 \mathrm{~m} \cdot \mathrm{y}$. (Nayior and others, 1970). The Bears Ears pluton of Nayior and others (1970) has been dated by the U-Pb zircon methods at $2,562 \pm 75 \mathrm{~m} \cdot \mathrm{y}$. This unit appears to be part of the Popo Agie batholith.

Oniy one intrusive event has been noted in the Owi Creek Mountains. Nkomo and others (1978) report a preliminary $\mathrm{Pb}-\mathrm{Pb}$ whole-rock age of 2,645 \pm $60 \mathrm{~m} . \mathrm{y}$. However, the geology is complex, and the existence of two intrusive events is not preciuded.

Geochronologic studies in the Hartville uplift are in progress at this time. Preliminary $\mathrm{Rb}-\mathrm{Sr}$ whole-rock resuits suggest an age of approximateiy 2,600 m.y. (Z. E. Peterman, oral communication, 1980).

Three separate whole-rock ages have been reported for the granite in the northern Laramie Range. The Rb-Sr resuits are 2,496 $440 \mathrm{~m} \cdot \mathrm{y}$. (Hiilis and Armstrong, 1974) and 2,512 $\pm 25 \mathrm{~m} \cdot \mathrm{y}$. (Johnson and Hilis, 1976). The reported $\mathrm{Pb}-\mathrm{Pb}$ age is 2,530 \pm 80 (Nkomo and others, 1979).

The Archean granites of central Wyoming have been affected by multiple postintrusive events. Each area has been intruded by diabase dikes. At least 
some of these are oniy siightiy younger than the granites (Peterman and Hijdreth, 1978), but two younger periods of dike intrusion may exist (Condie and others, 1969).

The southern portion of the area studied was affected by at least one middle Proterozoic event that reset $\mathrm{K}-\mathrm{Ar}$ and $\mathrm{Rb}-\mathrm{Sr}$ mineral ages (Peterman and Hiidreth, 1978). The resetting is interpreted to be the resuit of vertical tectonics. The Owi Creek Mountains and northern Wind River Range are not known to have been affected by this event; however, in these areas much of the exposed granite is ciose to the Cambrian weathering surface (Granger and others, 1971; Nkomo and others, 1978). A11 of the areas studied were uplifted and eroded during the Laramide event. 


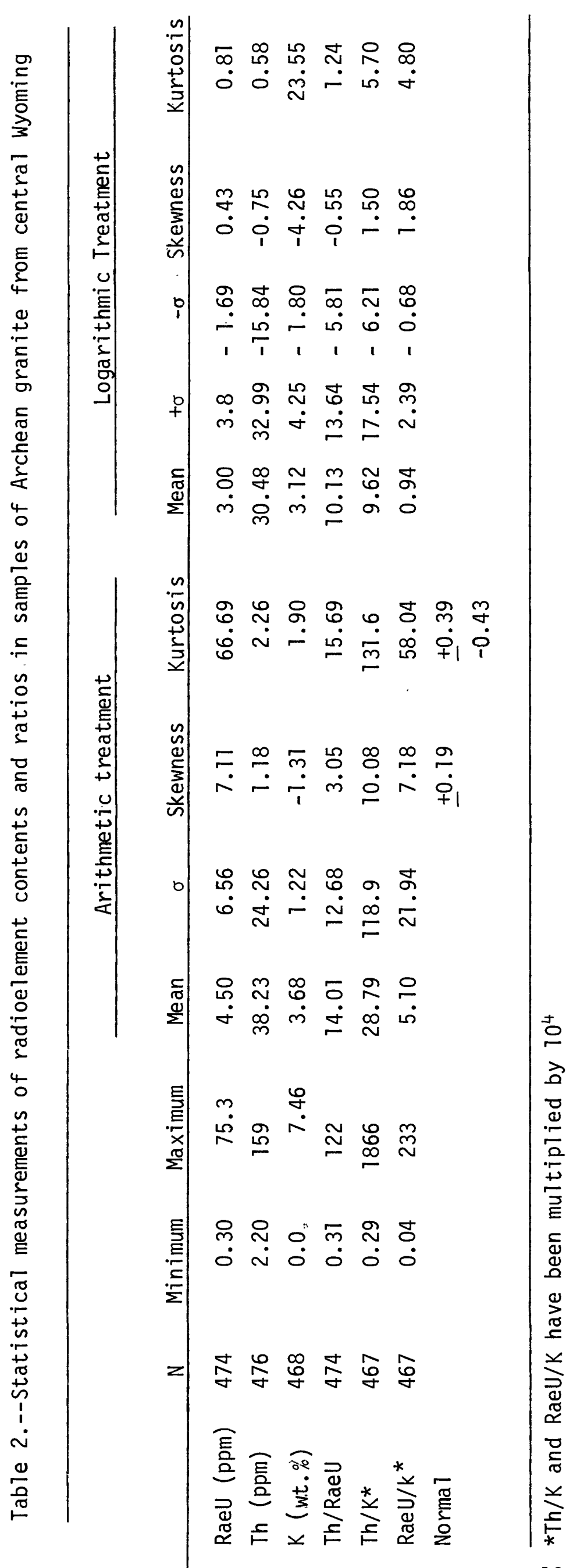




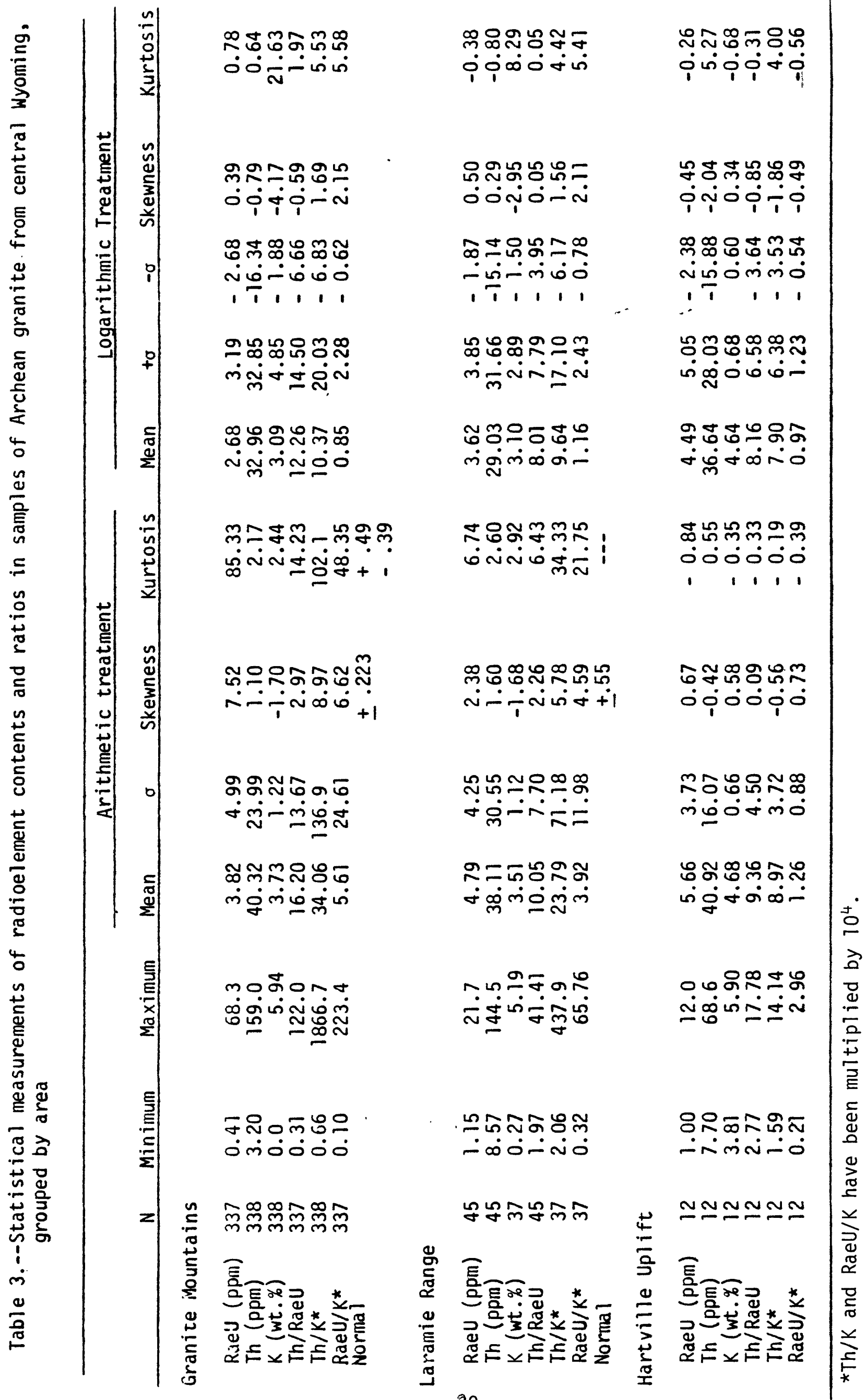




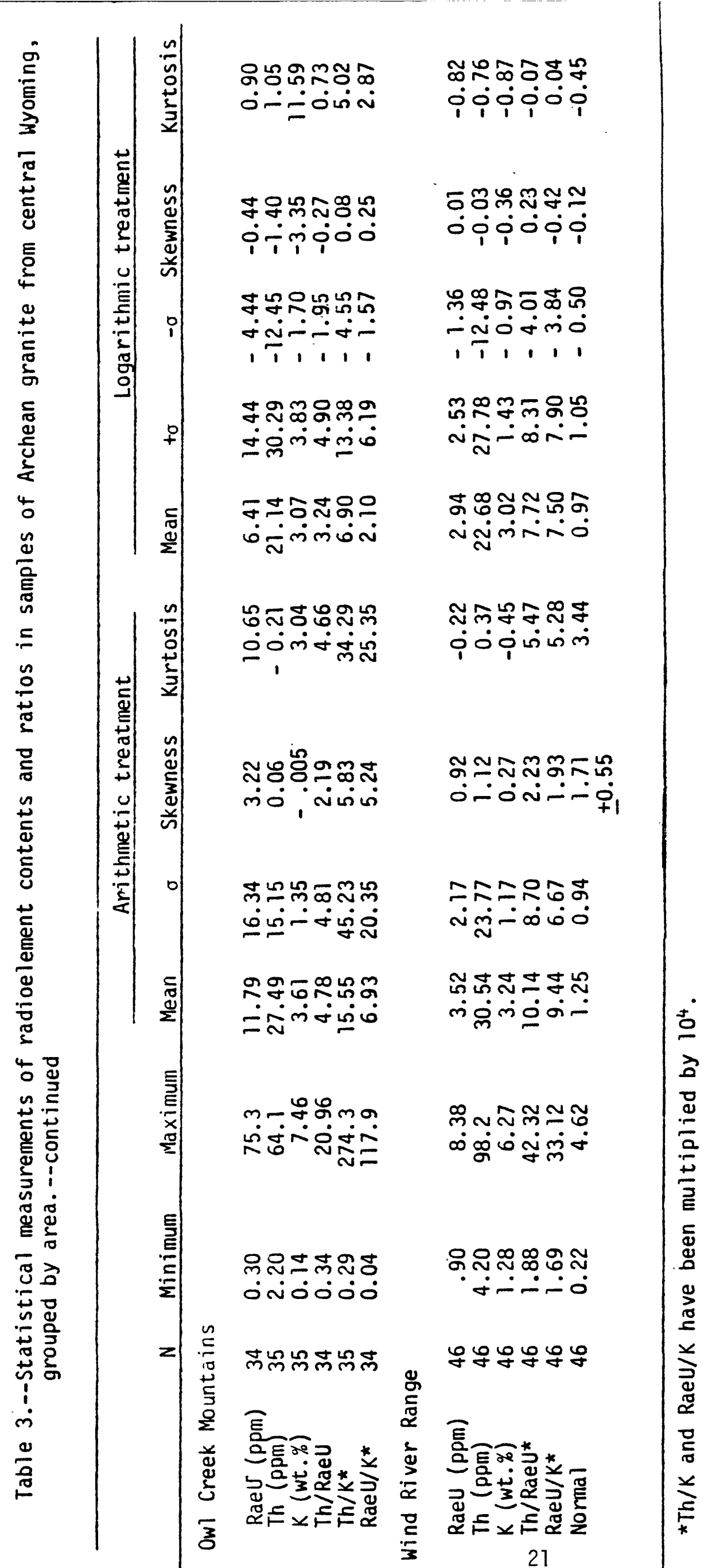




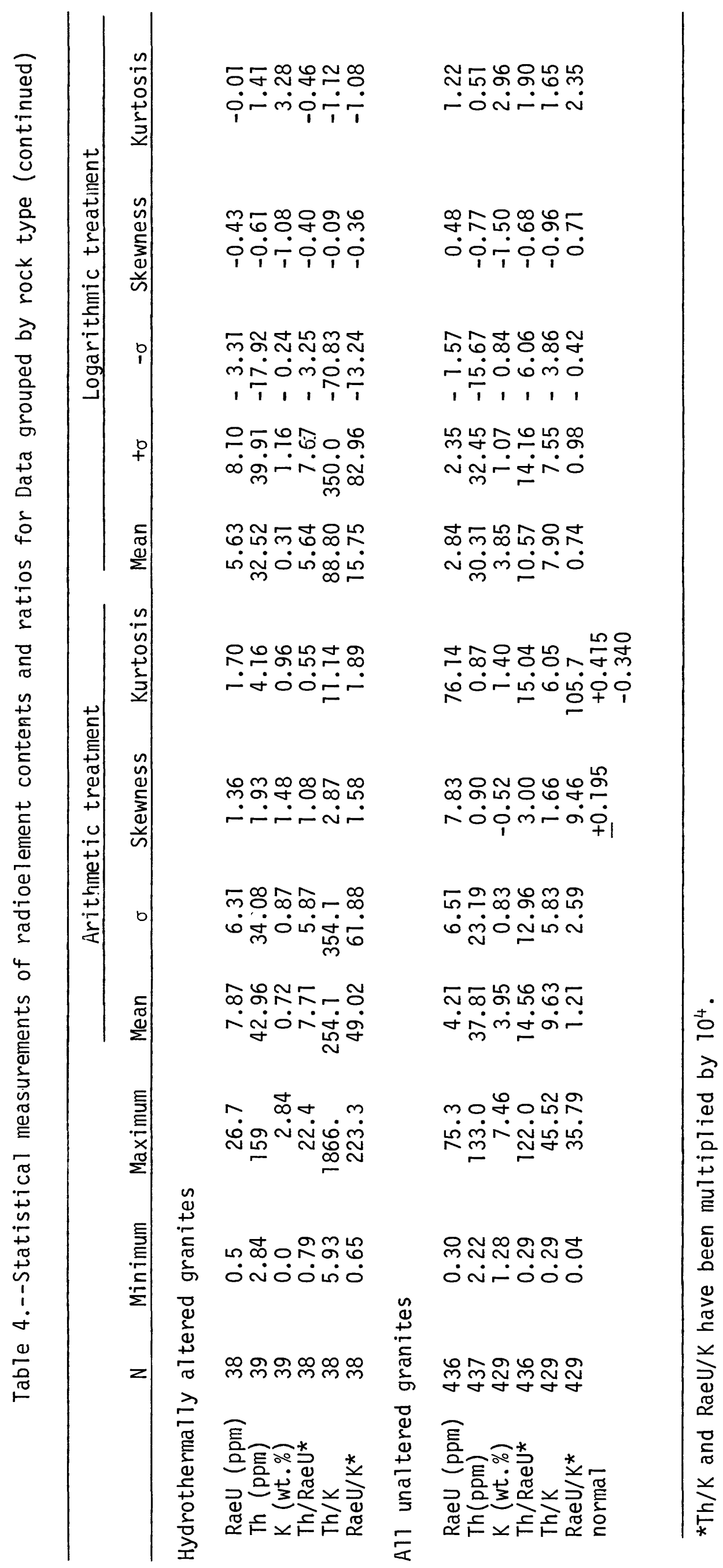




\section{RESULTS AND DISCUSSION}

The data for radioelement contents and ratios are presented in tabie 1 . The entire data set was evaluated statisticaliy using both arithmetic and logarithmic input (tabie 2). Subsets of the data grouped by area (tabie 3 ) and by rock type (tabie 4) were treated in a similar fashion.

In the total data set, and for each subset where there are enough data for a statisticaliy valid estimate, the uranium and thorium data and ratios involving these elements more closely approximate a lognormal distribution than a normal one. This tendency is indicated by the lower absolute values of skewness and kurtosis calcuiated in the logarithmic treatment relative to those of the arithmetic treatment (tabies 2, 3, and 4). The approximation of lognormal distributions is aiso shown graphicaliy for all of the thorium data and for all of the Th/RaeU ratios on figures 2 and 3 . Potassium data are better represented by a normal distribution; this is especialiy true if hydrothermaily aitered sampies are omitted from the statistical treatment (tabie 4). Subsequent discussion of the resuits wili therefore use the arithmetic mean and deviation for potassium and the geometric means and deviations for uranium and thorium and for ratios invoiving these two eiements.

In order to evaluate anomalies, we have compiled data for approximateiy 2,000 granitic samples (including those given in this report) for the contiguous United States. The arithmetic averages for uranium and thorium concentrations are not significantly different from those given by Rogers and Adams (1969 a,b), but our data base allows us to use geometric means and means of ratios rather than ratios of means. For our prei iminary U.S. data base, geometric means for uranium, thorium, Th/U, Th/K, and $U / K$ are $3.54 \mathrm{ppm}, 16.76$ $\mathrm{ppm}, 4.73,5.00 \times 10^{-4}$, and $1.05 \times 10^{-4}$ respectiveiy. The arithmetic mean for potassium (as K) is 3.59 weight percent. 


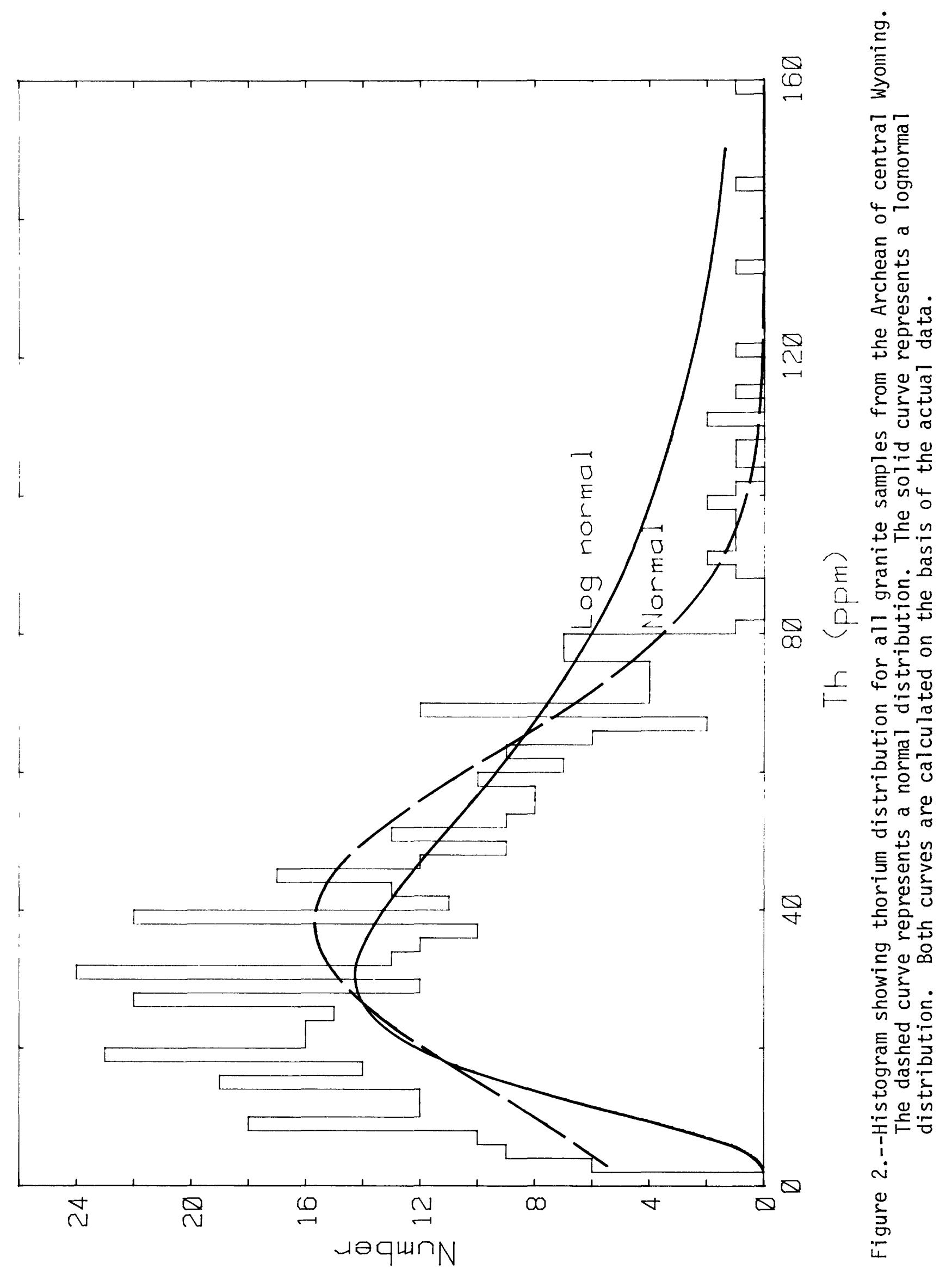




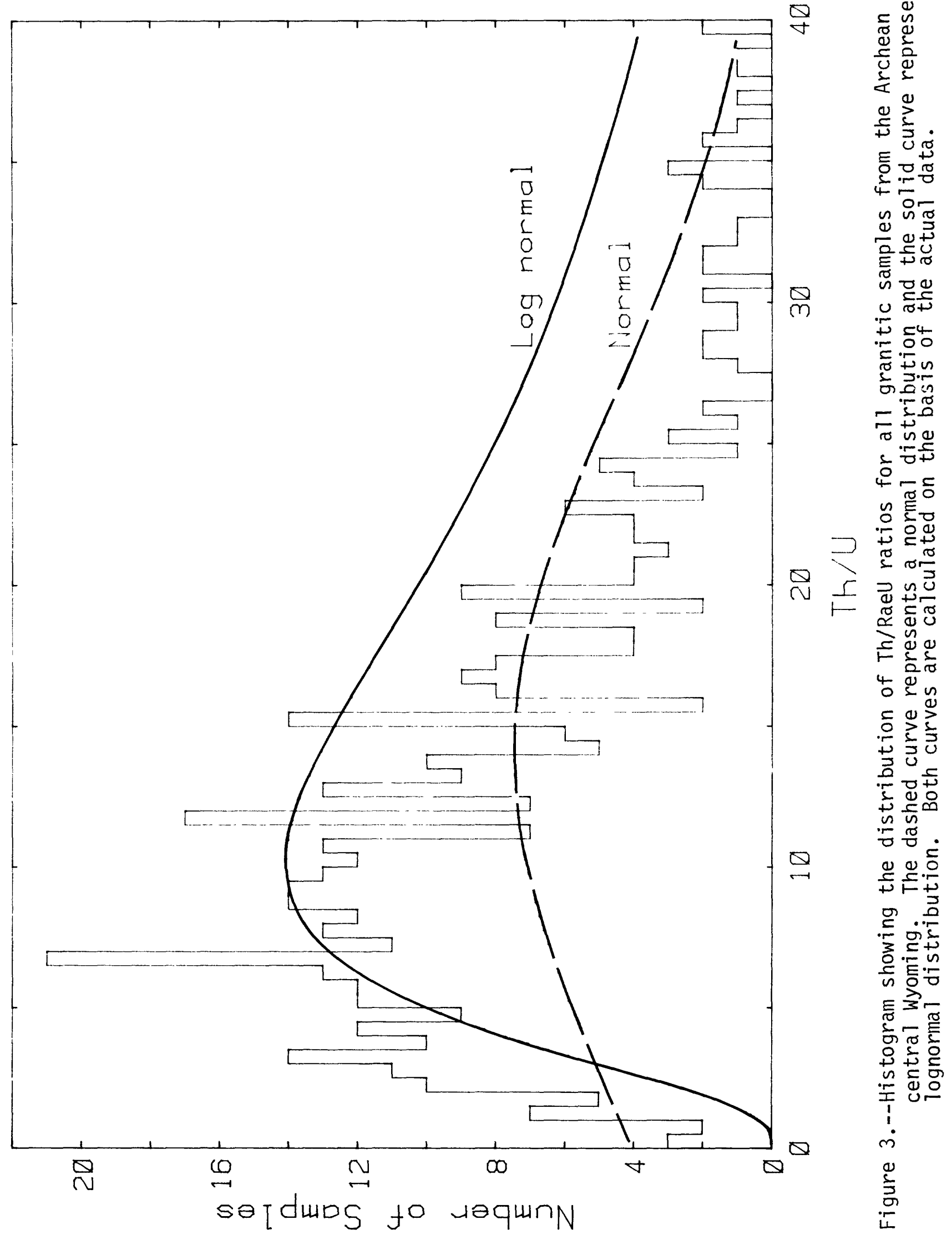


Subdividing the central Wyoming data on the basis of rock type (table 4) shows clear differences in radioelement contents and small differences in radioelement ratios. Relative to our U.S. data base, the biotite granites contain approximately normal amounts of uranium, but are greatly enriched in thorium. Consequentiy $\mathrm{Th} / \mathrm{U}$ and $\mathrm{Th} / \mathrm{K}$ ratios are anomalously high. The leucocratic granites have anomalousiy low uranium and thorium contents, but still exhibit a high Th/U ratio. The mafic granites contain approximateiy normal amounts of thorium and have sijghtly low uranium contents. Both Th/U and $T h / K$ ratios are somewhat high. The $U / K$ ratio for each type of unaltered granite is somewhat low reiative to our U.S. data base.

The hydrothermaily aitered granites are markediy depieted in potassium and slightiy high in uranium content, and they contain neariy twice as much thorium as the average U.S. granite. The resuiting ratios are nearly normal for Th/U and anomalousiy high for $T h / K$ and $U / K$. However, exclusion of these obviousiy atypical sampies from the total data set does not markedly change the means or statisticai parameters for uranium, thorium, or the Th/U ratio (tables 2 and 4 ). Thus, in order to retain as large a data base as possible for comparisons between areas, ail sampies will be used.

The relative proportions of uranium, thorium, and potassium for each of the sampied areas is compared to our U.S. data base on figure 4 by use of Iines that represent geometric means for radioelement ratios in the U.S. data base. For each of the five areas, most of the data plots to the left of the line that indicates a Th/K ratio of $5.0 \times 10^{-4}$. Thus all five areas exhibit high thorium contents relative to potassium.

Uranium concentrations relative to potassium are not generaily anomaious (fig. 4). In the Owi Creek Mountains there is a tendency towards high uranium contents relative to potassium, but these data are dominated by drili core 

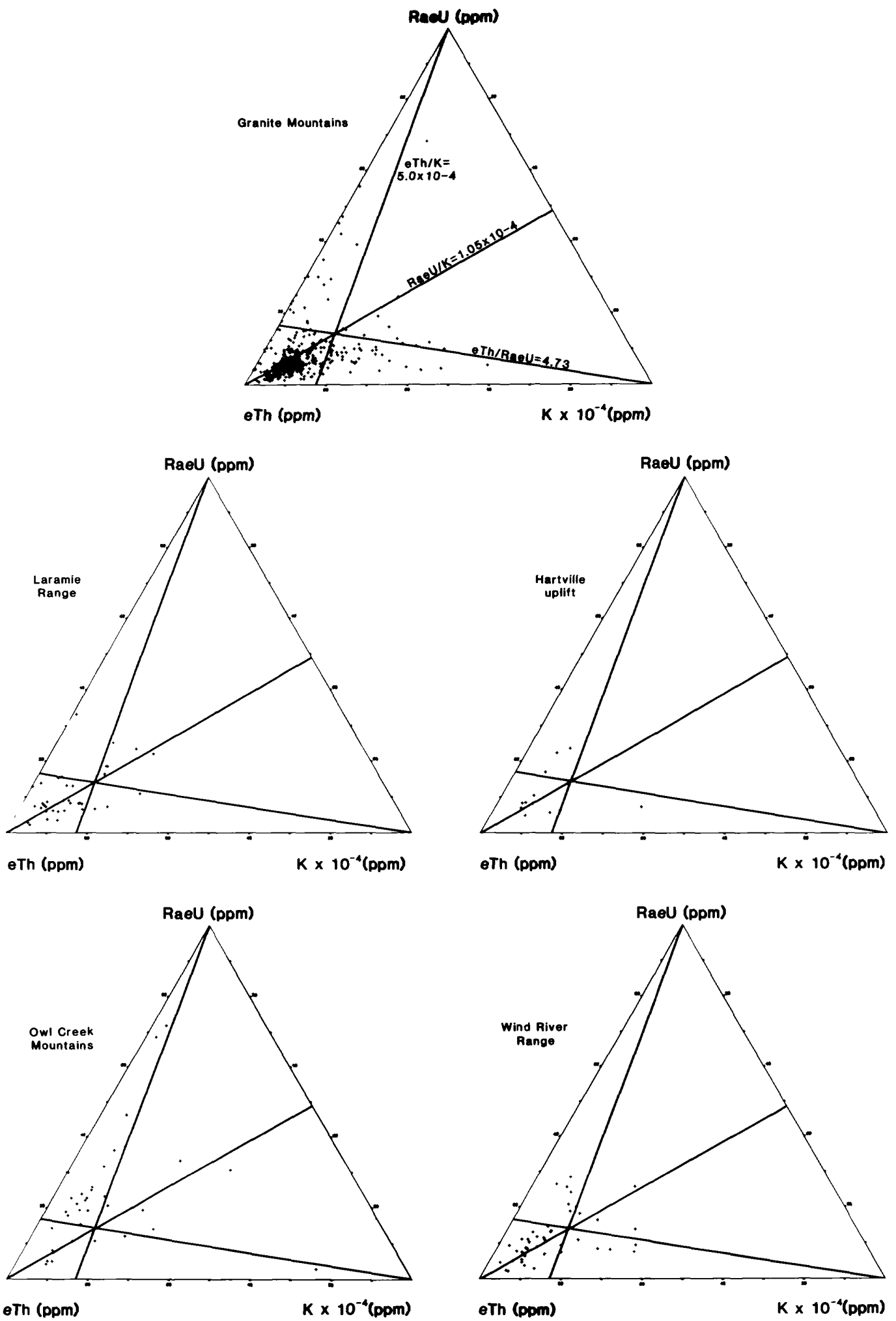

- Figure 4.--Ternary diagrams showing relative proportions of uranium (RaeU), thorium (eTh), and potassium $\left(K \times 10^{-4}\right)$ for each of the sampled areas. Lines representing geometric means for radioelement ratios in a data set of approximately 2000 granitic rocks are shown for comparison. 
samples (supplied by Rocky Mountain Energy) and therefore may not be representative of granite at the surface. Furthermore, the area drilled is mineralized, although obviousiy mineralized samples were excluded from the data sets. In the Granite Mountains and to a lesser extent in the Laramie Range, there is a tendency towards low concentration of uranium relative to potassium, but the geometric means are not significantiy different from the $1.05 \times 10^{-4}$ mean of our U.S. data base.

In each area, except the Owl Creek Mountains, most of the data plot below a line corresponding to a Th/U ratio of 4.73 (fig. 4 ). Thus, thorium contents are generaliy high relative to uranium. In the Owi Creek Mountains, high thorium reiative to uranium occurs only in some of the surface samples (Table 1).

Inasmuch as thorium contents are high relative to both uranium and potassium, the high $T h / U$ ratios in four of the five areas might be attributed to thorium enrichment, however isotopic data suggest that the high ratios are due to Tertiary uranium depietion. This is shown on figure 5 , where Th/U ratios calculated from thorogenic and uranogenic lead are compared to measured ratios. Implicit in the construction of this diagram is that the age of the granite and initial composition of lead are known and that lead has not been gained or lost. For most of the sampies, these conditions can be satisfied. To change the geometric mean Th/U ratio from 1.86 to 3.93 for the samples shown on figure 5 requires an average uranium loss of 60 percent. If the isotopic data are typical of the entire sample population, then the Th/RaeU ratio of 10.13 (table 2) indicates an average uranium loss of approximately 82 percent.

Aithough the Archean granitic rocks of central Wyoming are all enriched in thorium relative to a preliminary average for granitic rocks of the United 


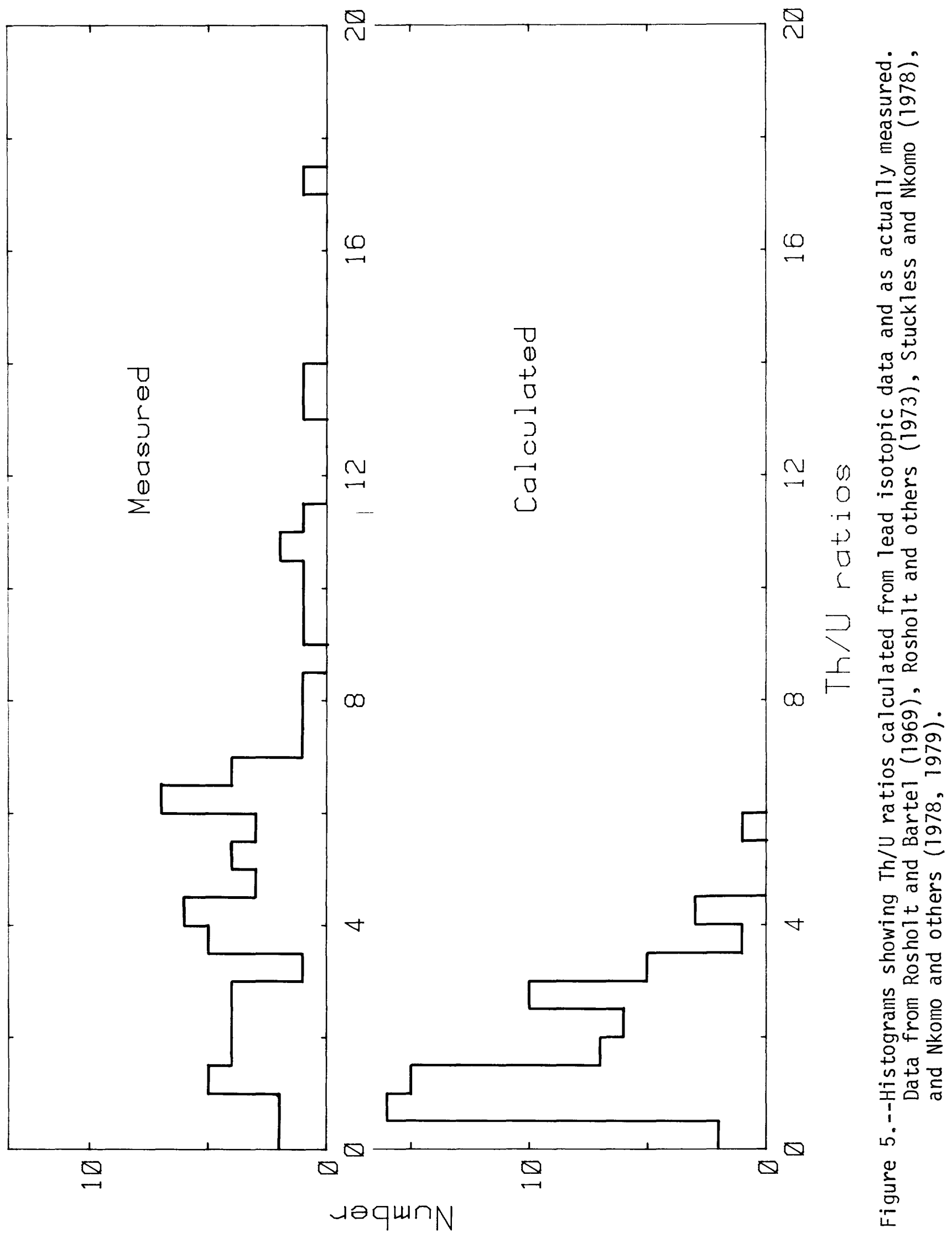


States, and all seem to have been enriched in uranium at one time, the Eocene paieodrainage reconstruction of Seeland (1976, 1978, and written communication, 1981) shows that ali but two of the Wyoming Tertiary uranium deposits are in sandstones whose provenance was the Granite Mountains or the Northern Laramie Range. We tentativeiy suggest that aithough all five areas contain granites that are similar in radioelement distribution, geologic differences may expiain the distribution of uranium ore.

Much of the granite in the Owi Creek Mountains, the Hartvilie uplift, and at least the northern part of the Wind River Range has not been deeply eroded during the Tertiary. Thus much of the currentiy exposed weathering surface was also exposed during the late Precambrian, and it is possibie that the granite in these areas lost leachabie uranium at that time. As a resuit, these granites would not have been able to provide much uranium to nearby Tertiary basins.

The dominant type of granite within a given area is another variable that may be highly significant in the determination of source-area favorability. Leucocratic and mafic granites currentiy contain much less thorium and uranium than biotite granite (tabie 4), and thus terrane dominated by these rock types may provide a much less favorable source. Much of the southern Wind River Range is dominated by the Louis Lake batholith, which is a mafic granitic rock (quartz diorite to granodiorite). Thus the apparent lack of uranium deposits in sediment with this provenance seems reasonable.

Silver (1976) suggested that uranium content of zircons might be an indicator of uranium provinces. By analogy, zircons with more than $1000 \mathrm{ppm}$ uranium may be an indicator of source-rock favorability. Zircons from the Louis Lake Batholith contain less than 500 ppm uranium (Nayior and others, 1970). In contrast, zircon from the spatialiy associated Bears Ears piuton 
contains more than 1500 ppm uranium (Naylor and others, 1970). Thus two independent lines of evidence suggest that the southern Wind River Range may contain both favorabie and unfavorabie source rock.

Zircon data are aiso avilabie for the Granite Mountains (Ludwig and Stuckiess, 1978). Two samples of the biotite granite of Lank in Dome and one of the hydrothermaily aitered equivaient contain zircons with uranium concentrations in excess of $1500 \mathrm{ppm}$. The eariier granite of Long Creek Mountain is a low thorium granite with relatively low Th/U ratios. Zircons from one sample of this unit contain an average of $850 \mathrm{ppm}$ uranium (Ludwig and Stuckiess, 1978).

In view of the correspondence of high Th/U ratios with large amounts of uranium loss and the correspondence of uraniferous zircons with high wholerock thorium contents, these radioelement characteristics are considered to be significant indicators of uranium provinces similar to that in centrai Wyoming. The distribution of high thorium contents and high Th/U ratios relative to the Eocene paieodrainage and known ore deposits is shown on figures 6 and 7. Aithough the high sample density in the Granite Mountains reiative to that in the other four areas tends to overemphasize this area, the proportion of sampies with more than twice the geometric mean of our U.S. data base is by far greatest in the Granite Mountains for both thorium contents and Th/U ratios. The iowest proportion of anomaious sampies is in the southern Wind River Range. Approximately one third of the sampies from the northern Laramie Range are anomaious in terms of both thorium contents and Th/U ratios. This area and the Granite Mountains (which were deeply eroded in the Tertiary) are the provenance for the sandstones that host uranium ore. 
Figure 6.- Map of the central Wyoming region showing distribution of samples with anomalous thorium contents (small open squares), the distribution of Tertiary uranium deposits (Butler 1972), and the Eocene drainage pattern (Seeland, 1976, 1978, and written communication, 1981). Smali squares indicate thorium contents greater than $39 \mathrm{ppm}$; pluses indicate thorium contents between 39 and $16.5 \mathrm{ppm}$; dots indicate thorium contents less than $16.5 \mathrm{ppm}$. Data are gridded to a 2-minute grid and piotted points represent averages of all data within a 2-minute area. The large soidd squares represent uranium districts with more than $1,000,000$ tons of ore. Solid circles represent deposits with 1,000 to $1,000,000$ tons of ore, and open circles represent deposits with less than 1,000 tons of ore. 


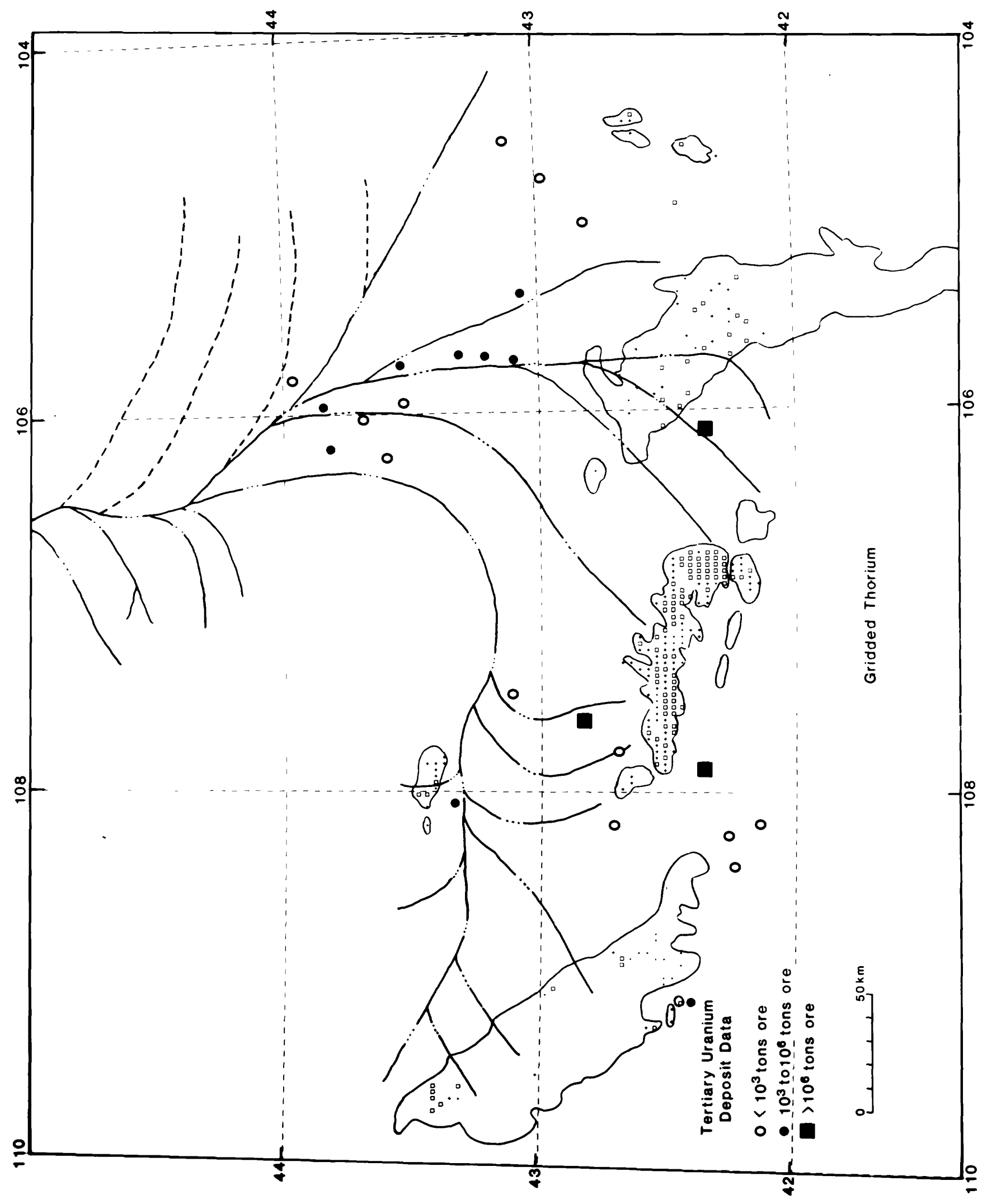


Figure 7.- Map of the centrai Wyoming region showing distribution of samples with anomaious $T h / U$ ratios (smail open squares), the distribution of Tertiary uranium deposits (Butier, 1972) and the Eocene drainage pattern (Seeland, 1976, 1978, and written communication, 1981). Sma1i squares indicate Th/U ratios greater than 11.8; pluses indicate a Th/ $U$ ratio between 5.1 and 11.8 ; dots indicate a Th/U ratio less than 5.1. Data are gridded to a 2-minute grid, and plotted points represent averages of all data within a 2minute area. See added description on Fig. 6. 


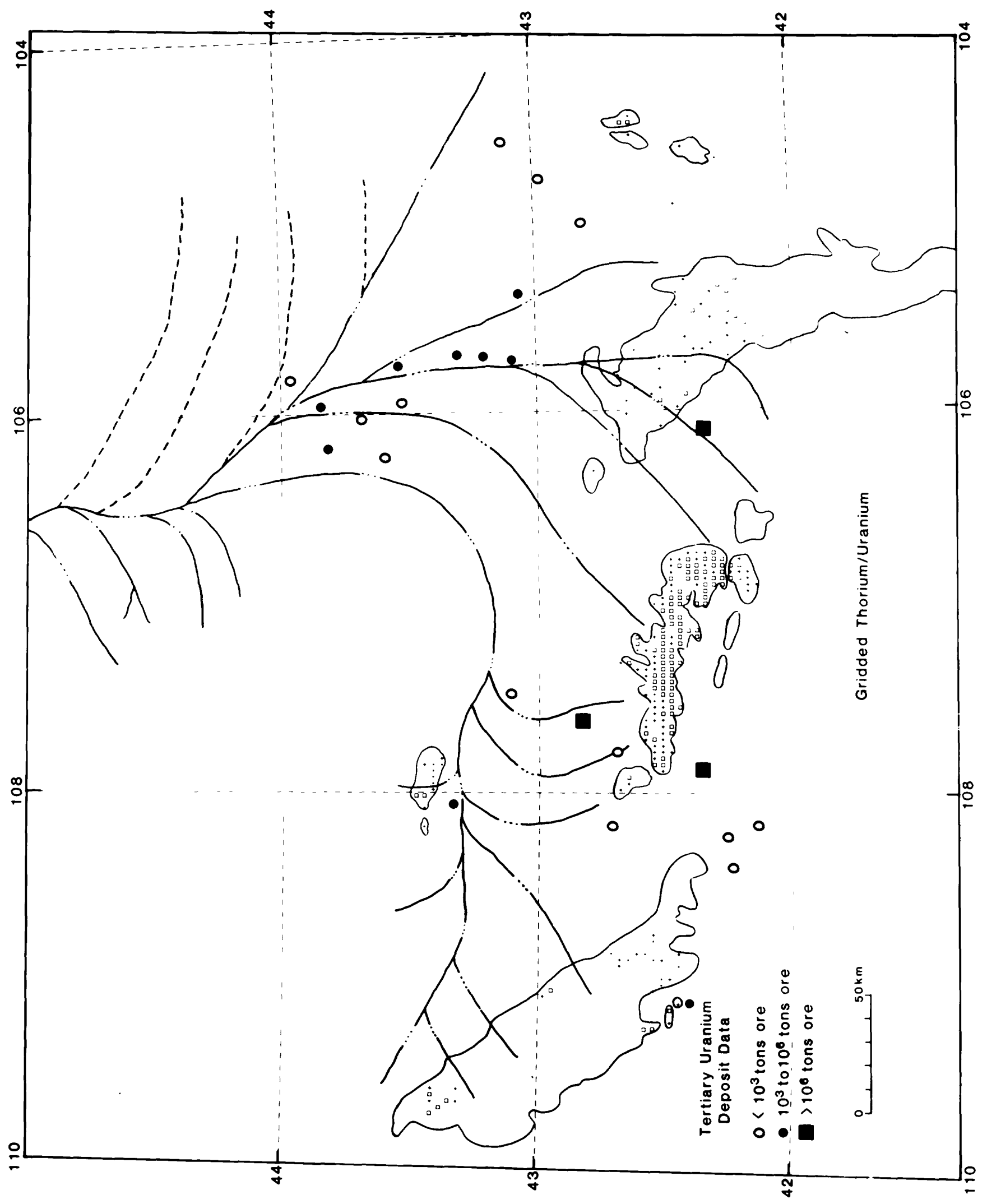




\section{SUMMARY}

The Archean granites of central Wyoming are characterized by anomalousiy high thorium contents and high Th/U ratios. This is especially true of granites in the provenance region for the Tertiary sandstones that host uranium ore. In the areas for which the high $T h / U$ ratios can be assessed, isotopic data indicate that Tertiary uranium loss caused the ratios to change from anomalously low (<2) to anomalously high ( 10$)$.

The Hartville uplift, Owl Creek Mountains, and northern Wind River Range are associated closely, with only small uranium deposits. Granites in the latter two areas tend to be less anomalous in terms of thorium content and Th/U ratios (Table 3). Furthermore, much of the granite in all three regions is close to the Cambrian weathering surface and may have lost most of its leachable uranium prior to the deposition of the mineralized Tertiary sandstones.

The southern Wind River Range is more deeply eroded; however, much of the granitic rock in this region is fairly mafic. This type of granite does not seem to be particulariy anomalous in terms of thorium content or Th/U ratio. Thus the apparent lack of large uranium deposits in sandstones with this provenance is not surprising.

Finally, the statistical distribution of uranium and thorium concentrations and ratios involving these elements is approximated more closely by a lognormal distribution than by a normal one. Work is in progress to see if log-normal distribution is typical for these variables for granitic rocks in general. 


\section{References}

Bassett, W. A., and Giletti, B. J., 1963, Precambrian ages in the Wind River Mountains, Wyoming: Geological Society of America Bulletin, v. 74, p. 209-212.

Bayley, R. W., 1965a, Geologic map of the Miners Delight quadrangle, Fremont County, Wyoming: U.S. Geological Survey Quadrangle Map GQ-460.

-..-- 1965b, Geologic map of the Louis Lake quadrangle, Fremont County, Wyoming: U.S. Geological Survey Quadrangle Map GQ-461.

Bunker, C. M., and Bush, C. A., 1966, Uranium, thorium, and radium analyses by gamma-ray spectrometry (0.184-0.352 million electron volts), in Geological Survey research 1966: U.S. Geological Survey Professional Paper 550-B, p. B176-B181.

-.-- 1967, A comparison of potassium anaiyses by gamma-ray spectrometry and other techniques, in Geological Survey research, 1967: U. S. Geological Survey Professional Paper 575-B, p. B164-B169.

Butler, A. P., Jr., 1972, Uranium, in Mallory, W. W., ed., Geologic atlas of the Rocky Mountain region: Denver, Rocky Mountain Association of Geologists, p. 315-317.

Condie, K. C., 1969, Petrology and geochemistry of the Laramie batholith and related metamorphic rocks of Precambrian age, eastern Wyoming: Geological Society of America Bulletin, v. 80, p. 57-82.

Condie, K. C., Leech, A. P., and Baadsgaard, H., 1969, Potassium-argon ages of Precambrian mafic dikes in Wyonming: Geological Society of America Builetin, v. 80 , p. 899-906.

Granger, H. C., McKay, E. J., Mattick, R. E., Patten, L. L., and MacIlroy, Paul, 1971, Mineral resources of the Glacial Primitive Area, Wyoming: U.S. Geological Survey Bulletin 1319-F, 113 p. 
Hills, F. A., and Armstrong, R. L., 1974, Geochronology of Precambrian rocks in the Laramie Range and implications for the tectonic framework of Precambrian southern Wyoming: Precambrian Research, v. 1, p. 213-225. Johnson, R. C., and Hills, F. A., 1976, Precambrian geochronology and geology of the Boxelder Canyon area, northern Laramie Range, Wyoming: Geological Society of America Bulletin, v. 87, p. 809-817.

Ludwig, K. R., 1978, Uranium daughter migration and U-Pb isotope apparent ages of uranium ores, Shirley Basin, Wyoming: Economic Geology, v. 73, p. 2949.

.... 1979, Age of uranium mineralization in the Gas Hills and Crooks Gap Districts, Wyoming as indicated by U-Pb isotope apparent ages: Economic Geology, v. 74, p. 1654-1668.

Ludwig, K. R. and Stuckiess, J. S., 1978, Uranium-lead isotope systematics and apparent ages of zircons and other minerals in Precambrian granitic rocks, Granite Mountains, Wyoming: Contributions to Mineralogy and Petrology, v. 65, p. 243-254.

Nayior, R. S., Steiger, R. H., and Wasserburg, G. J., 1970, U-Th-Pb systematics in $2700 \times 10^{6}$-year old plutons from the southern Wind River Range, Wyoming: Geochimica et Cosmochimica Acta, v. 34, p. 1133-1159. Nkomo, I. T., and Rosholt, J. N., 1972, A lead-isotope age and U-Pb discordance of Precambrian gneiss from Granite Mountains, Wyoming: U.S. Geological Survey Professional Paper 800-C, p. C169-C177.

Nkomo, I. T., Stuckiess, J. S., Thaden, R. E., and Roshoit, J. N., 1978, Petrology and uranium mobility of an eariy Precambrian granite from the Owi Creek Mountains, Wyoming, in Wyoming Geological Association, 30th Annual Field Conference Guidebook: p. 335-348. 
Nkomo, I. T. Roshoit, J. N., and Dooley, J. R., Jr., 1979, U-Th-Pb systematics in surface and drili core samples of a Precambrian basement rock from Laramie: Earth Science Bulletin, v. 12, no. 4, p. 1-i4.

Pearson, R. C., Kitisgaard, T. H., Patten, L. L., and Mattich, R. E., 1971, Mineral resources of the Popo Agie Primitive Area, Fremont and Sublette Counties, Wyoming: U.S. Geological Survey Bulletin 1353-B, 55 p. Peterman, Z. E., and Hildreth, R. A., 1978, Reconnaissance geology and geochronology of the Precambrian of the Granite Mountains, Wyoming: U.S. Geological Survey Professional Paper 1055, 22 p.

Rogers, J. J. W., and Adams, J. A. S., 1969a, Uranium, in K. H. Wedepohl, ed., Handbook of geochemistry, v. 2, no. 4, Berlin, Springer-Verlag, p. 92-B to $92-0$.

--- 1969b, Thorium, in K. H. Wedepoh1, ed., Handbook of Geochemistry, v. 2, no. 4, Berlin, Springer-Veriag, p. 90-1 to 90-0.

Roshoit, J. N., and Bartel, J. A., 1969, Uranium, thorium, and lead systematics in the Granite Mountains, Wyoming: Earth and Planetary Science Letters, v. 7, p. 141-417.

Roshoit, J. N., Zartman, R. E., and Nkomo, I. T., 1973, Lead isotope systematics and uranium depletion in the Granite Mountains, Wyoming: Geological Society of America Bulletin, v. 84, p. 989-1002.

Seeland, D. A., 1976, Relationship between early Tertiary sedimentation patterns and uranium mineralization in the Powder River Basin, Wyoming: Wyoming Geological Association, 28th Annual Field Conference, 1979, Guidebook, p. 53-64.

---- 1978, Sedimentology and stratigraphy of the lower Eocene Wind River Formation, central Wyoming: Wyoming Geological Association, 30th Annual Field Conference, 1978, Guidebook, p. 181-198. 
Silver, L. T., 1976, A regionai uranium anomaly in the Precambrian basement of the Colorado Plateau (abs.): Geological Society of America Abstracts with Programs 1976, p. 1107-1108.

Snyder, G. L., 1980, Map of Precambrian and adjacent Phanerozoic rocks of the Hartvilie uplift, Goshen, Niobrara, and Piatte Counties, Wyoming: U.S. Geologicai Survey Open-File Report 80-779, $11 \mathrm{p}$.

Steiger, R. H., and Jager, E., 1977, Subcommission on geochronoiogy:

Convention on the use of decay constants in geo- and cosmochronology:

Earth and Pianetary Science Letters, v. 36, p. 359-362.

Stuckiess, J. S., Bunker, C. M. VanTrump, George, Jr., and Bush, C. A, 1978, Radiometric resuits and areal distribution for granitic sampies from the Granite Mountains, Wyoming: U.S. Geologicai Survey Open-File Report 78$803,51 \mathrm{p}$.

Stuckiess, J. S., and Nkomo, I. T., 1978, Uranium-lead isotope systematics in uraniferous aikaii-rich granites from the Granite Mountains, Wyoming: implications for uranium source rocks: Economic Geology, v. 73, p. 427441 .

Thaden, R. E., 1976a, Preliminary geologic maps of the De Pass quadrangie, Fremont and Hot Springs Counties, Wyoming: U.S. Geologicai Survey OpenFile Report 76-207.

-.-- 1976b, Preliminary geoiogic map of the Guffy Peak quadrangle, Fremont and Hot Springs Counties, Wyoming: U.S. Geologicai Survey Open-File Report 75-229.

-.-- 1976c, Preiiminary geologic map of the Birdseye Pass quadrangle, Fremont and Hot Springs Counties, Wyoming: U.S. Geologicai Survey Open-File Report 76-346. 
Tourtelot, H. A., 1953, Geology of the Badwater area, central Wyoming: U.S. Geological Survey $0 i 1$ and Gas Investigations Map OM-C124.

VanTrump, George, Jr., and Miesch, A. T., 1977, The U.S. Geological Survey RASS-STATPAC system for management and statistical reduction of geochemicai data: Computers and Geosciences, v. 3, p. 475-488.

Wenner, D. B., Stuckless, J. S., and Chang, K. K., 1981, Constraints on the origin of a granitic uranium source rock, Granite Mountains, Wyoming (Abs.): American Association of Petroleum Geologists Bulletin, v. 65, p. 573. 